\title{
Research on Wheel-Rail Local Impact Identification Based on Axle Box Acceleration
}

\author{
Yuanjin Ji (iD, Junwei Zeng, and Wenjing Sun \\ Institute of Rail Transit, Tongji University, Shanghai, China \\ Correspondence should be addressed to Yuanjin Ji; jiyuanjin@tongji.edu.cn
}

Received 18 January 2022; Revised 26 January 2022; Accepted 29 January 2022; Published 25 February 2022

Academic Editor: Traian Mazilu

Copyright ( 2022 Yuanjin Ji et al. This is an open access article distributed under the Creative Commons Attribution License, which permits unrestricted use, distribution, and reproduction in any medium, provided the original work is properly cited.

\begin{abstract}
Abnormal vibration signals of tramcar are mostly nonstationary and nonlinear signals. This study applied the Hilbert-Huang transform (HHT) to analyze abnormal vibration of the tramcar, aiming to overcome the limitations of some traditional timefrequency analysis methods, such as Fourier transform, in dealing with nonstationary and nonlinear signals. Additionally, to address mode aliasing produced during empirical mode decomposition (EMD) used in classical HHT, this study proposed to first use complete EMD with adaptive noise for the decomposition of original vibration data, then eliminate the trend-term components with the calculated correlation coefficients, and finally perform denoising on high-frequency noisy components using the wavelet threshold method. After weighted reconstruction using denoised high-frequency components and low-frequency information components, data processing was finally optimized via HHT. Taking a tramcar as an example, the Hilbert spectra of the vertical acceleration of axle box were plotted via HHT, and the time-instantaneous, frequency-instantaneous energy $3 \mathrm{D}$ relations were obtained for the location of impact points. Further, the vibration characteristics were analyzed and quality indexes were calculated in combination with the marginal spectra so as to judge the reasons for abnormal vibration and failure modes of the tramcar. The results revealed that the proposed method was feasible and effective in vibration feature extraction and vibration impact analysis for tramcars.
\end{abstract}

\section{Introduction}

The 100\% low-floor light-rail vehicles in the urban railway system, owing to no need of platforms, low floor through the whole car, and great convenience in boarding and alighting, have gradually gained popularity in various big cities. Compared with high-speed railways, tramcars are still subjected to abnormal vibration induced by some structural diseases, mainly including ordinary joint cracks and faulting of slab ends during the operation process. Figure 1 shows the vibration anomalies of the axle box induced by the line impact and the impact and fatigue damage to the axle bearing.

In-depth investigation of abnormal vibration data is of great significance, which contributes to analyzing the failure mode of the vehicle-line system. Scholars all over the world have conducted a great deal of research on the effects of rail irregularity on the vehicle's dynamic characteristics. Xu et al. selected representative and actual rail irregularity set from various amounts of data and established the probability model of rail irregularity based on the time-frequency transform [1]. The probability transfer relation between rail anomalies and system response could be addressed by introducing the probability density evolution method. Xu et al. also proposed an inversion model of rail irregularity and inversed true geometric profile of rail based on vehicle's vibration amplitude, wavelength, and probability under different rail regularity conditions [2]. Gao et al. established the theoretical model of some typical steel rail weld irregularities based on the analysis of the surface geometrical shape of steel rail in the weld zone. They validated the established rail weld unevenness model in the dynamic valuation of high-speed railway weld unevenness by measuring and simulating the wheel-rail dynamic response in the case of uneven railway welds. Moreover, they investigated the effects of different steel rail weld unevenness on 


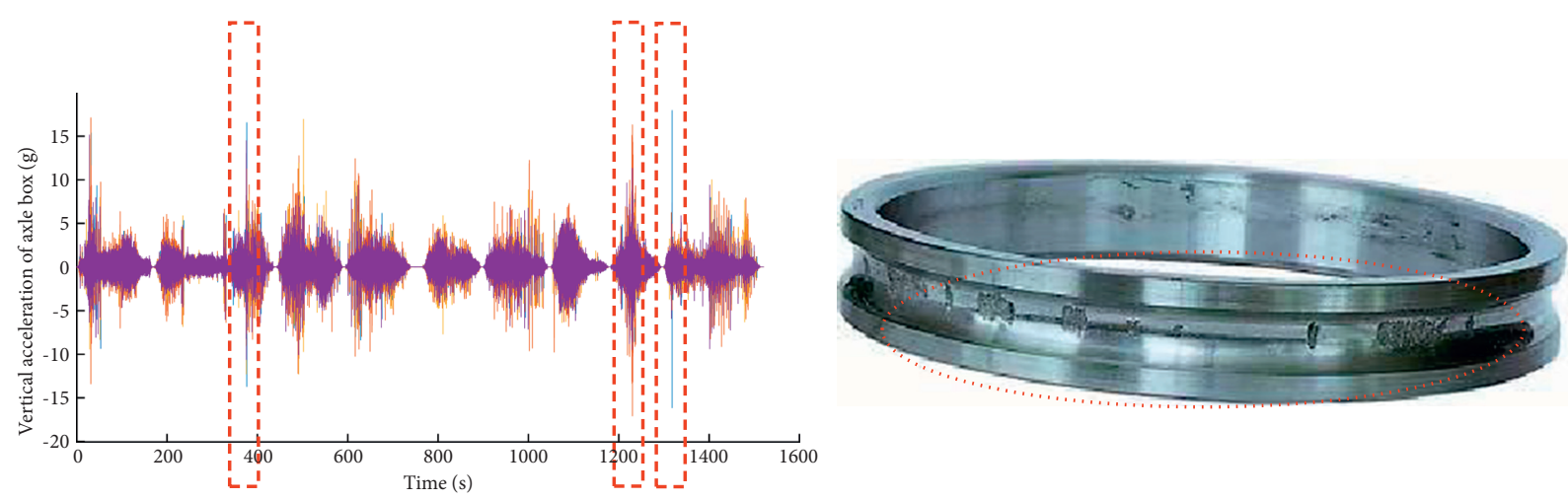

FIgURE 1: Some abnormal vibration phenomena.

wheel-rail dynamic interaction based on the vehicle-rail coupled dynamic model and the established steel rail weld unevenness model [3]. Xu et al. investigated the amplitudefrequency curves that described the relationship between rail irregularity and the vibration response of the coupled system and the distribution characteristics of sensitive wavelengths of rail irregularity. They introduced the wavelength weight coefficient to rail irregularity and proposed a novel rail regularity evaluation index-rail weighted quality index [4]. Yang et al. analyzed the vibration characteristics of the gear case on high-speed trains under the action of wheel-rail excitement and evaluated the vibration characteristics of the gear case of different wheels [5]. Niu et al. performed the related simulation for accurately analyzing the rail irregularity spectra and analyzed the sources of the vibrationexcited load using the wheel-rail coupled dynamic model. Moreover, they analyzed the characteristics of vibrationexcited load [6]. Xiao et al. proposed a Kalman filtering algorithm based on vehicle dynamic response that could identify rail irregularities of railway bridges by considering vehicle-bridge (VB) interaction in real time, aiming to identify rail irregularities. Further, rail irregularities could be identified with an optimal estimation of the state vectors of the VB system [7]. Using dynamic simulation, Xiao et al. analyzed the variation rules of the vibration characteristics of the gear system on high-speed trains under excitation of rail irregularities. They concluded that lateral, longitudinal, and vertical vibrations of the gear system could be enhanced in the case of excitation of rail irregularities, among which the variation amplitude of the vertical vibration acceleration was the greatest [8].

The axle box of a train is in direct and rigid connection with the wheel sets. The acceleration signal of the axle box reflected the external turbulence induced by rail irregularities on the vehicle's dynamics. Therefore, many scholars proposed the evaluation methods of rail irregularities based on the acceleration of the axle box. Grassie et al. expounded the advantages of the evaluation methods based on the acceleration of the axle box on rail irregularities in terms of economic benefits and operational convenience [9]. Based on wavelet analysis, Caprioli et al. proposed the method of extracting the frequency characteristics of rail irregularities from the acceleration data of the axle box [10]. Molodova et al. proposed the method of detecting rail surface defects based on the scale-averaged wavelet power [11]. Based on the principle of generalized resonance, Xiong Kang et al. employed resonance demodulation for converting the analysis of low-frequency periodic impact characteristics of steel rail-welded joint to vehicle-railway system into the analysis of high-frequency resonance. They proposed the evaluation method of high-speed railway welded joint based on the acceleration of the axle box [12]. Liu et al. proposed the rail impact index based on the vertical acceleration of the axle box after band-pass filtering, which could be used for evaluating short-wave irregularities of high-speed railway [13].

Many time-domain or frequency-domain analysis methods exist for analyzing the vibration signals of tramcars. However, some traditional joint time-frequency analysis methods represented by Fourier transform showed certain limitations in dealing with complex failure signals. For a tramcar, the abnormal vibration signal is generally composed of nonstationary and nonlinear data. The Hilbert-Huang transform (HHT) showed particularly superiorities in the processing of this kind of signals. HHT took signal decomposition based on its own time-scale characteristics without presetting any basic functions, which was essentially different from the Fourier decomposition and wavelet decomposition. HHT could accurately reveal the internal characteristics of the data, with high resolution in time and frequency domains, which also showed great significance and extensive applications in some domains, including vibration analysis, fault detection, and parameter identification. Chen et al. proposed an improved empirical mode decomposition (EMD) method that effectively extracted the dynamic characteristics of the vehicle-rail coupled system [14]. Li et al. performed the time-frequency analysis on vertical vibration of vehicle-rail system with HHT and quantitative research on the relationship between rail irregularities and the vibrational acceleration of the vehicle in the vertical direction [15]. Su et al. applied HHT to bridge vibration analysis and dealt with the vibration response when the vehicle passed a bridge, providing an adequate tool for bridge vibration analysis and health detection [16]. Li et al. proposed a new noise reduction technology of fully integrated empirical mode decomposition of underwater acoustic signals based on adaptive noise, minimum mean square variance criterion, and minimum 
mean square adaptive filter [17]. Chen et al. proposed particle swarm optimization least squares support vector machine [18] Babouri et al. proposed a hybrid method based on adaptive noise fully integrated empirical mode decomposition, optimized wavelet multiresolution analysis, and Hilbert transform [19].

Wheel flat scars are a kind of wheel tread damage, such as fatigue failures, abrasion, scratches, and exfoliation of wheel treads, that commonly form in the actual operation of railway locomotives. The rotating wheels with flat scars could generate intermittent pulse turbulence on wheel tracks during the traveling process. The generated impact was several times or a dozen times greater than the ordinary impact, thereby leading to the vibration of running parts of the vehicle, the steel rails, and underrail structures [20]. Newton et al. equalized flat damage on wheels as a kind of rail irregularity and pointed out that the flat-scar impacts were equivalent to rail uneven joints in dynamics [21].

This study focused on tramcars and proposed a rail local irregularity evaluation method based on the acceleration of the axle box. The complete ensemble EMD with adaptive noise (CEEMDAN) was proposed for the decomposition of the original signal so as to overcome modal aliasing problems existing in the EMD of traditional HHT. Then, the correlation coefficients of various intrinsic mode function (IMF) components after decomposition with the original signal were calculated, while the trend-term components with low correlation coefficients were eliminated. After wavelet threshold denoising, high-frequency noisy components were combined with main information components for weighted reconstruction. Hilbert transform was then performed on the reconstructed components so as to obtain the Hilbert spectra of the vibration signal. Time-frequency-instantaneous energy images were plotted, while the moment and frequency band information of abnormal vibration were obtained. Further, the impact points were located via the speed integral. For each impact region, the Hilbert integral was individually performed so as to obtain the marginal spectrum of the vibration signal. Based on the marginal spectrum, the main frequency of abnormal vibration was determined and the quality index of each impact was also calculated for analyzing the line problems corresponding to the abnormal vibration regions and the failure modes. This study adopted the vertical acceleration signal of the axle box in the simulation model test as the original signal and effectively extracted abnormal vibration characteristics using the aforementioned methods, which provided effective information for identifying failure modes. Furthermore, the feasibility of the proposed method in the rail joint impact identification and analysis was experimentally validated.

\section{Improved CEEMDN-HHT Analysis}

Traditional time-frequency methods mainly adopted the kurtosis value, mean square value, effective value, probability density function, and wavelet analysis to judge abnormal vibration.
Table 1 lists the features and limitations of the aforementioned traditional rail irregularity evaluation methods/ indexes.

Figure 2 shows the kurtosis value, wavelet analysis, probability density, and root-mean-square images of the abnormal vibration signal of the axle box induced by the irregularities of a rail segment. Both the kurtosis value and the effective value could show the position and degree of the impact points in time domain but could not reflect the characteristic frequencies of the excited abnormal vibration. Wavelet analysis could analyze the vibration signal in both time and frequency domains; however, the signal around the abnormal impact point was featured by typical nonlinearity and nonstationarity, and wavelet transforms performed poorly in the feature extraction of this kind of signals. Under the wheel-rail excitation in the rail impact form, the aforementioned traditional methods showed great limitations and failed in adequately extracting the characteristic information of abnormal vibration around the impact position.

2.1. CEEMDAN Algorithm. CEEMDAN is an improved mode decomposition algorithm based on EMD, which also introduces Gaussian noise and multisuperposition averaging for noise cancellation. Using CEEMDAN, the adaptive Gaussian white noise signal via EMD was added in each phase of solving IMF. After obtaining each order of IMF components, white noise was added to the residual value so as to obtain the mean IMF and take successive iterations until complete signal decomposition occurred. CEEMDAN could not only effectively address the mode aliasing problem in EMD [19] but also overcome the transfer of white noise from high to low frequency.

The principle of the CEEMDAN algorithm has been described as follows.

After adding the positive and negative auxiliary white noises in pairs to the original signal $x(t)$, EMD decomposition was performed on the novel signal so as to derive the first-order IMF component $c_{1}(t)$. Taking the average of $n$ IMF components produced in $n$ iterations, the first-order IMF component of CEEMDAN could be obtained:

$$
\overline{c_{1}(t)}=\frac{1}{N} \sum_{i=1}^{n} c_{1}^{i}(t) .
$$

By subtracting $\overline{c_{1}(t)}$ from the original signal $x(t)$, a new series could be obtained. The aforementioned steps were then repeated until the residual signal component $r_{n}$ was a periodic function, a monotonic function, a constant, or a simple signal function only with an extremum. Using CEEMDAN, the original signal was decomposed as follows:

$$
x(t)=\sum_{k=1}^{K} \overline{c_{k}(t)}+r_{k}(t),
$$

where $c_{i}$ denotes each IMF component after decomposition and includes different components from high- to low-frequency bands and $r_{k}(t)$ denotes the residual signal. 
TABLE 1: Comparison among traditional rail irregularity evaluation methods/indexes.

\begin{tabular}{|c|c|c|}
\hline Evaluation method/index & Feature & Limitations \\
\hline Kurtosis value & Time domain & $\begin{array}{l}\text { Lack of the extraction of frequency characteristics of } \\
\text { abnormal vibration }\end{array}$ \\
\hline Effective value & Time domain & $\begin{array}{c}\text { Lack of the extraction of frequency characteristics of abnormal } \\
\text { vibration }\end{array}$ \\
\hline $\begin{array}{l}\text { Probability density } \\
\text { function }\end{array}$ & Time domain & $\begin{array}{c}\text { Lack of the extraction of frequency characteristics of abnormal } \\
\text { vibration }\end{array}$ \\
\hline Wavelet analysis & $\begin{array}{l}\text { Time domain and frequency } \\
\text { domain }\end{array}$ & $\begin{array}{c}\text { Lack of the extraction of frequency characteristics of abnormal } \\
\text { vibration }\end{array}$ \\
\hline
\end{tabular}
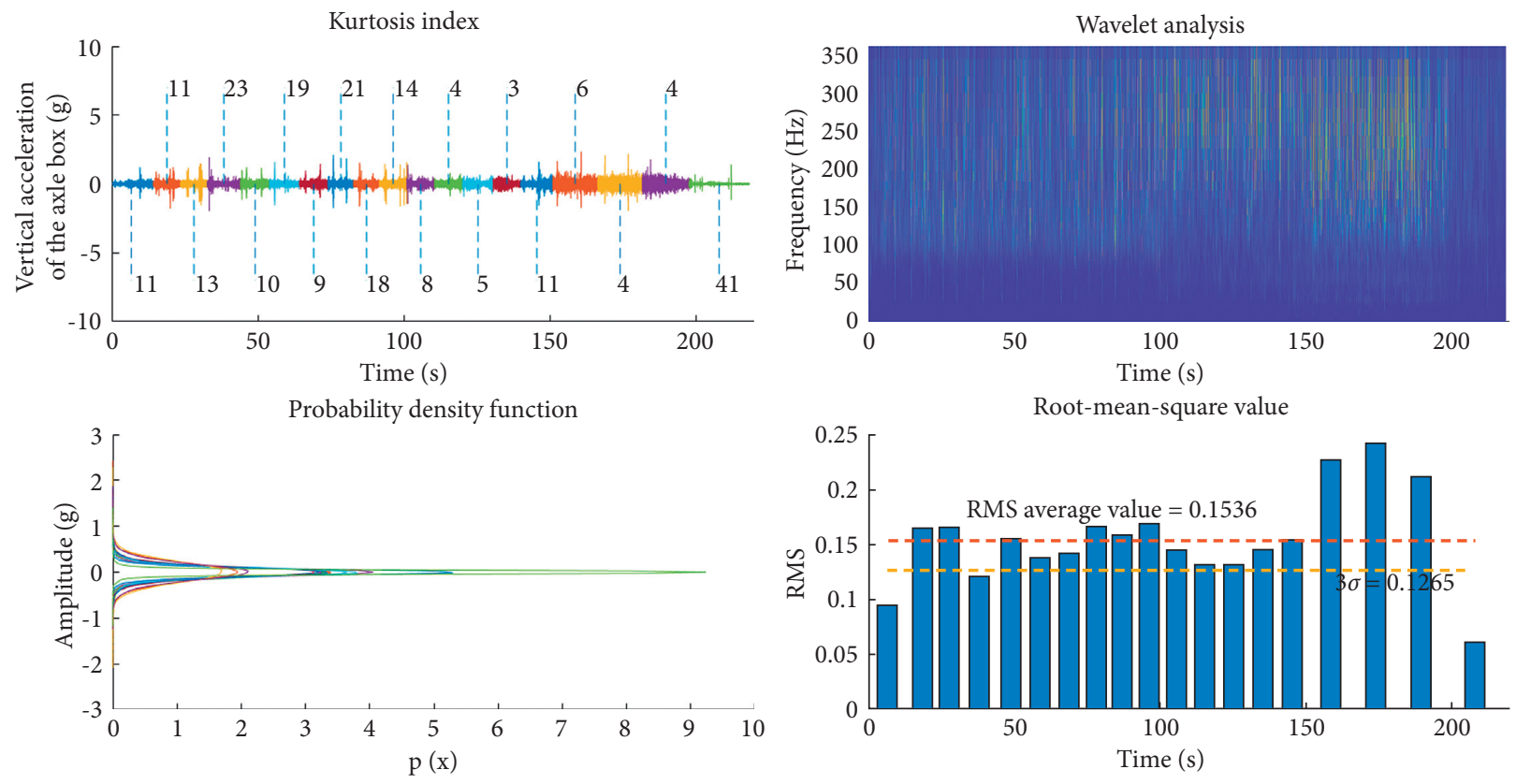

FIgURE 2: Traditional vibration analysis methods.

Contrary to the EMD algorithm, the used CEEMDAN algorithm showed great advantages in extracting the characteristics of the impact signal of railway vehicles.

The original signal shown in Figure 3 is selected as a simple example. Traditional HHT adopted EMD for data pretreatment. The implementation of EMD first helped identify the local extrema of the current signal. The envelope curves of local minimum and local maximum could be obtained by cubic spline curve so as to plot the mean curve.

The target signal of rail irregularities generally included some abnormal events such as impact and noise, which inevitably affected the selection of extrema. Therefore, the envelope obtained via EMD was regarded as the combination of a local envelope of abnormal events and the envelope of the real signal. Accordingly, the decomposed IMF components included a natural vibration mode of signal and abnormal events or contained the natural vibration modes at the neighboring characteristic time scales, thereby resulting in mode aliasing. Therefore, by combining CEEMDAN and HHT, the CEEMDAN-HHT impact signal feature extraction method was proposed in this study. CEEMDAN was used for replacing EMD, and the superiorities of CEEMDAN in rail irregularity research were validated via comparison.
EMD and CEEMDAN were performed on the aforementioned signal for mode decomposition. Figure 4 shows the first eight main components using different methods. As the local extrema showed multiple jumping changes within an extremely short interval in the EMD process, the mode aliasing phenomenon could be observed, as shown in Figure 4(a). Considering the IMF4 via EMD as an example, IMF4, as an IMF, included the characteristic time scales with a great difference. The noise was almost mixed in each IMF component via EMD, which reduced the significance of each individual IMF. Figure 4(b) shows the decomposition results with CEEMDAN. The IMF components were all found to be independent without mode aliasing. To be specific, IMF1 and IMF2 components were high-frequency noisy components, which could be denoised or eliminated in specific studies. The IMF3-IMF8 components could well reflect the characteristics of impact signal and serve as main information components of investigating the characteristics of impact signal. The IMF components via the CEEMDAN algorithm were purer than the results using EMD, suggesting that CEEMDAN was more suitable for extracting and investigating various characteristics of the signal. The further effects on the generated Hilbert spectra via HHT using two 


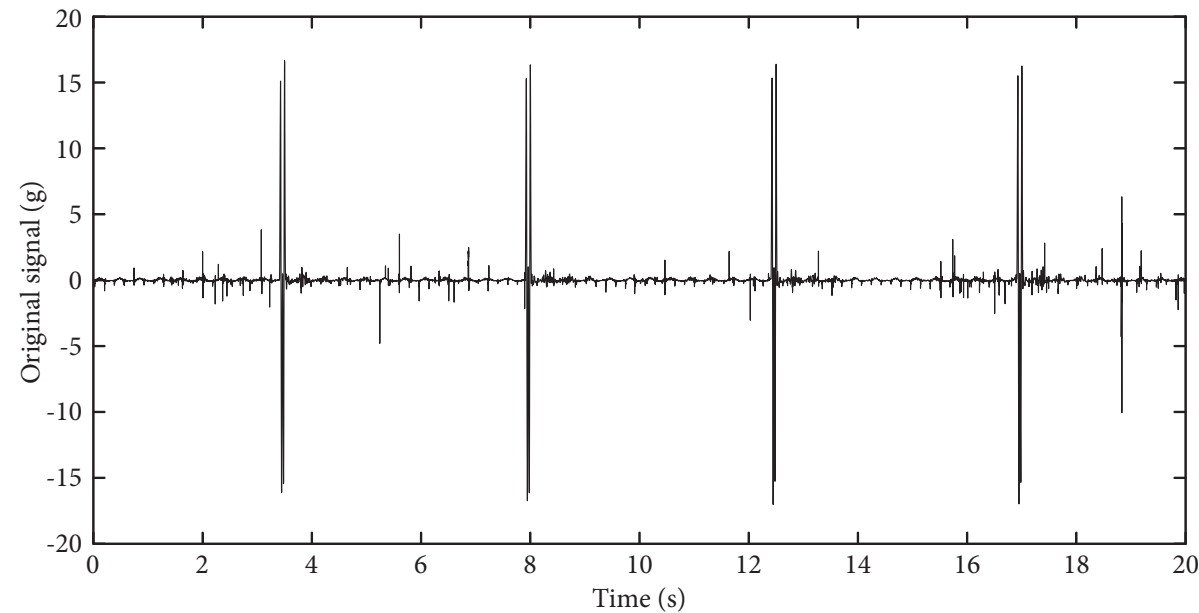

FIgURE 3: Original signal.
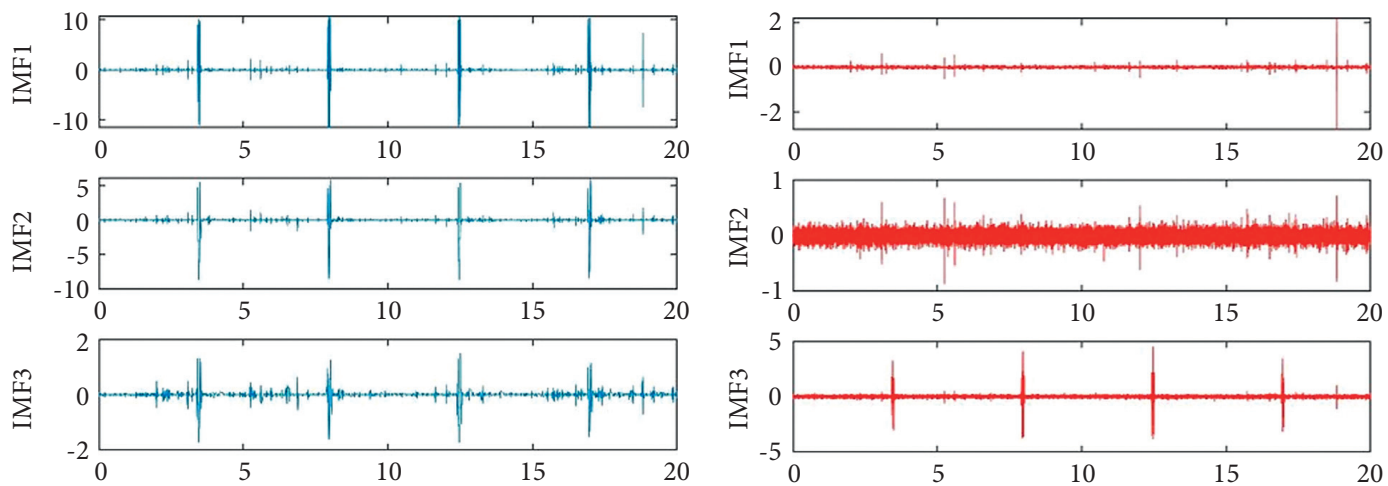

$\sum_{-0.5}^{5} \begin{array}{r}0.5 \\ 0\end{array}$
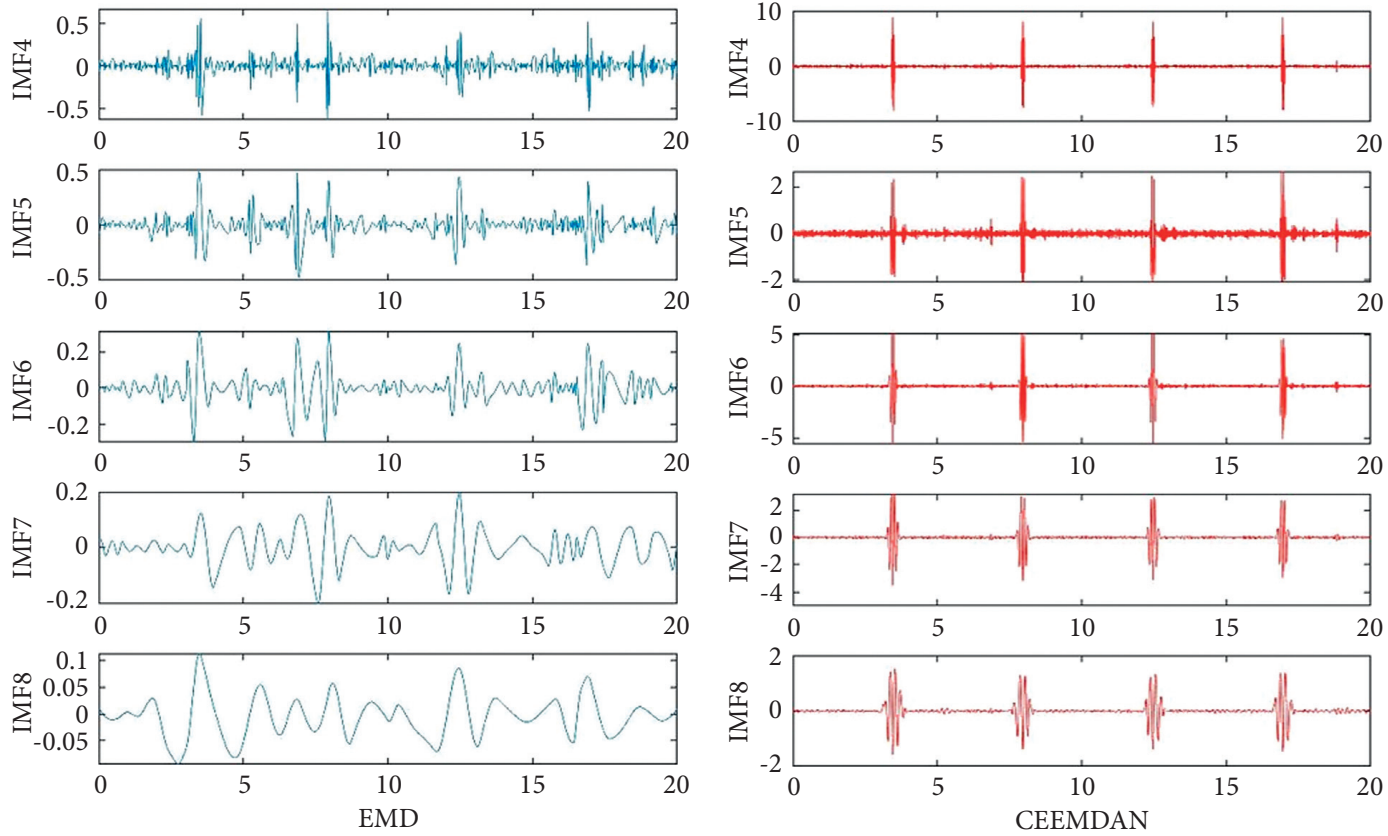

(a)

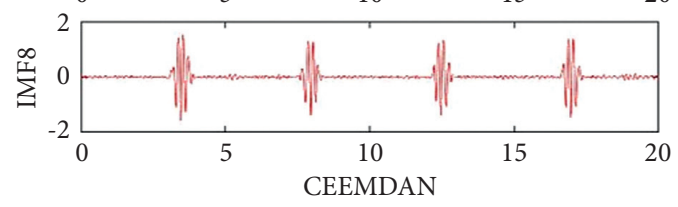

(b)

FIgURE 4: Decomposition results of the abnormal signal using (a) EMD and (b) CEEMDAN. 
different decomposition methods are described in detail in Section 4.1.

2.2. Hilbert-Huang Transform. HHT is appropriate for dealing with nonstationary and nonlinear signals, which is essentially a stationary processing technique for nonstationary signals. Using HHT, the fluctuations at different scales in the signal or trend terms could be gradually decomposed into various IMF components. Then, HHT was performed on various IMF components to derive the Hilbert spectra in both time and frequency domains. Finally, the frequency contents of the original signal could be characterized by instantaneous frequency and instantaneous energy.

The detailed procedures are described as follows. First, the Hilbert transform was performed on each IMF component $c_{i}(t)$ :

$$
d_{i}(t)=\frac{1}{\pi} \int_{-\infty}^{+\infty} \frac{c_{i}(\tau)}{t-\tau} \mathrm{d} \tau
$$

The analytical signal was established as follows:

$$
\begin{aligned}
z_{i}(t) & =c_{i}(t)+j d_{i}(t) \\
& =a_{i}(t) e^{j \varphi_{i}(t)} .
\end{aligned}
$$

The instantaneous amplitude of the corresponding IMF component was expressed as follows:

$$
a_{i}(t)=\sqrt{c_{i}^{2}(t)+d_{i}^{2}(t)}
$$

The corresponding phase was expressed as follows:

$$
\phi_{i}(t)=\arctan \frac{d_{i}(t)}{c_{i}(t)} .
$$

The instantaneous frequency could be further obtained as follows:

$$
\begin{aligned}
f_{i}(t) & =\frac{1}{2 \pi} w_{i}(t) \\
& =\frac{1}{2 \pi} \frac{\mathrm{d} \varphi_{i}(t)}{\mathrm{d} t} .
\end{aligned}
$$

Therefore, the original signal $x(t)$ could be rewritten as follows:

$$
x(t)=\operatorname{Re} \sum_{i=1}^{n} a_{i}(t) e^{j \int \omega_{i}(t) \mathrm{d} t},
$$

where Re denotes the real part. Equation (8) characterizes the time-frequency-amplitude 3D relation of $x(t)$. The distribution of signal amplitude on the time-frequency plane was referred to as the Hilbert spectrum $H(w, t)$ and expressed as follows:

$$
H(w, t)=\operatorname{Re} \sum_{i=1}^{n} a_{i}(t) e^{j \int \omega_{i}(t) \mathrm{d} t} .
$$

The Hilbert marginal spectrum $h(w)$ could thus be obtained by taking the integral of $H(w, t)$ :

$$
h(w)=\int_{0}^{T} H(w, t) \mathrm{d} t
$$

where $T$ denotes the overall length of the signal. The marginal frequency reflected the overall amplitude of instantaneous frequency in the signal, which could measure the contribution of each frequency to the amplitude within the time range and the accumulative amplitudes of all data lengths in statistics.

$H(w, t)$ could accurately describe the variation rules of signal amplitude with time and frequency in the whole frequency band, while $h(w)$ reflected the variation of signal amplitude in the whole frequency band.

2.3. Quality Index $q$. All impact positions in the range were segmented into several 0.2 -s intervals. The marginal spectrum could thus be obtained via HHT and the integral. The main amplitude at the impact point could be obtained from the marginal spectrum. Considering the fact that impacts always exist in the actual vibration signal, the rail impact quality index was introduced for evaluating the impact degrees at different positions. The impact points at which the quality index exceeded the threshold value could thus be judged as abnormal impact points. The rail impact quality index $Q$ was defined as follows:

$$
Q_{i}=\frac{A_{i}(\omega)}{\bar{A}(\omega)}, \quad(i=1,2,3, \ldots, n)
$$

where $A_{i}(\omega)$ denotes the main amplitude at the $i$ th impact point and denotes the mean value of main amplitudes et al.l impact points within the range.

2.4. Analysis Process. Figure 5 illustrates the analysis process of the abnormal vibration signal. First, the original signal was decomposed by CEEMDAN, and all IMF components were obtained. The correlation coefficients between various IMF components and the original signal were calculated. The trend-term components with low correlation were then eliminated. High-frequency components with noise were denoised with the wavelet threshold method. After the denoising, high-frequency components, main information components, and trend-term components were used for reconstruction in the weighted pattern. After the Hilbert transform, the Hilbert spectra with time and frequency joint distribution were plotted. The impact moments were identified and positioned based on the Hilbert spectra, while the impact positions were located via the speed integral. All impact areas were selected for the Hilbert transform. The marginal spectra were calculated via the integral, and the quality index was calculated so as to determine the location of the most serious impact point.

\section{Simulation for Validation}

The Universal Mechanism (UM) dynamic model of the tramcar was established to validate the effectiveness of the proposed method, as shown in Figure 6. The model consisted of two vehicle bodies, three bogies, four swing bolsters, six 


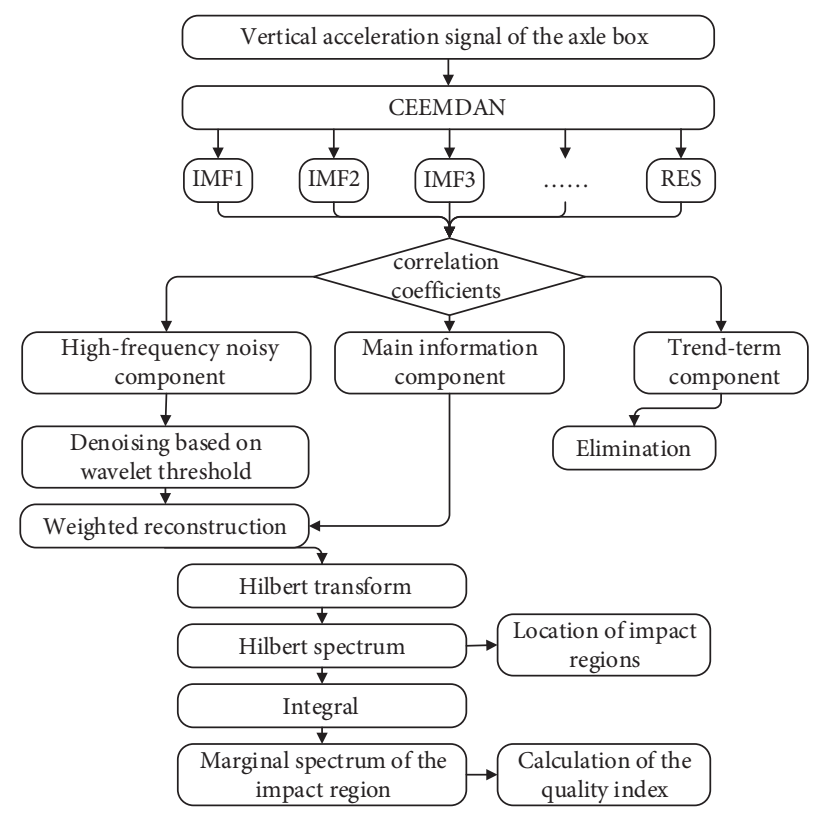

Figure 5: Flowchart of the algorithm.

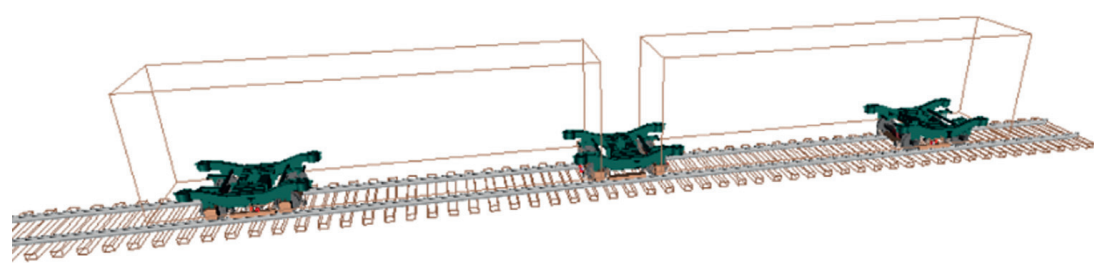

Figure 6: Established dynamic model for simulation.

axle bridges, and six independent wheel sets, as the main vehicle parameters of the model shown in Table 2 .

Local irregularities were superposed into the traditional six-level spectrum to simulate the bumping impact on the rail joint. Further, the first peak was 1.5 times greater than the subsequent peaks so as to distinguish the joint quality, as shown in Figure 7. Figure 8 shows the vertical acceleration data of the axle box when the vehicle moved at a speed of $20 \mathrm{~km} / \mathrm{h}$. The maximum acceleration appeared at the first peak and could be up to $25 \mathrm{~g}$, which was consistent with the rules of the preset rail spectrum.

First, CEEMDAN was performed on the abnormal vibration signal of the axle box of the tramcar, and a total of 18 IMF components were obtained. Among these, the first eight components (denoted as IMF1-IMF8) are shown in Figure 9.

The correlation coefficients between various IMF components and the original signal are listed in Table 3. Apparently, IMF5-IMF11 components were highly correlated with the original signal and could be regarded as the main components of the original vibration signal, which were concentrated with the most significant characteristics of the original signal and imposed the greatest effect on the overall response. The correlation coefficients between the other components and the original signal were all less than 0 . The IMF1-IMF4 components were high-frequency noisy components and so denoised using the wavelet threshold method. The IMF12-IMF18 components were trend-term components and had no significance to feature the extraction of the original signal and hence eliminated.

Accordingly, the IMF1-IMF4 components after denoising and IMF5-IMF11 were selected as information components for reconstruction. Figure 10 compares the original signal and the reconstructed signal and shows that the two signals coincided well. The main characteristics of the original signal could be retained.

By performing instantaneous processing on the information components of the vertical vibration signal of the axle box, instantaneous frequency, instantaneous phase, and instantaneous energy could be obtained so as to further plot the Hilbert spectrum of the vibration signal, as shown in Figure 11. Energy-concentrated amplification could be observed within the frequency bands of $15-30 \mathrm{~Hz}$ at $3.4 \mathrm{~s}$, $7.9 \mathrm{~s}, 12.4 \mathrm{~s}$, and $16.9 \mathrm{~s}$. The instantaneous energy at these moments far exceeded the values at the other moments and in the other frequency bands. Four obvious impacts at a frequency of approximately $20 \mathrm{~Hz}$ could be observed at these moments during the whole vibration process.

Figure 12 visually displays the time-instantaneous, frequency-instantaneous energy $3 \mathrm{D}$ relation of the vibration signal. Apparently, four impacts existed in this range at an average interval of approximately $2.3 \mathrm{~s}$. To be specific, the first impact at $3.465 \mathrm{~s}$ was the most obvious, whose energy reached the peak at $20 \mathrm{~Hz}$ with a maximum instantaneous 
TABLE 2: Vehicle parameters.

\begin{tabular}{lcc}
\hline Parameters & Definition & Value \\
\hline$m_{c}$ & Mass of each vehicle body & $10,000 \mathrm{~kg}$ \\
$m_{s 1}$ & Mass of the swing bolster on the end bogie & $500 \mathrm{~kg}$ \\
$m_{s 2}$ & Mass of the swing bolster on the middle bogie & $250 \mathrm{~kg}$ \\
$m_{b}$ & Mass of each truss & $1,800 \mathrm{~kg}$ \\
$m_{w}$ & Mass of each wheel set & $800 \mathrm{~kg}$ \\
$L$ & Central distance between two bogies & $10 \mathrm{~m}$ \\
$D$ & Longitudinal distance between the mass center of the vehicle and the center of the bogie & $0.5 \mathrm{~m}$ \\
$A$ & Axle distance between bogies & $1.9 \mathrm{~m}$ \\
$S$ & Offset distance of the center plate & - \\
\hline
\end{tabular}
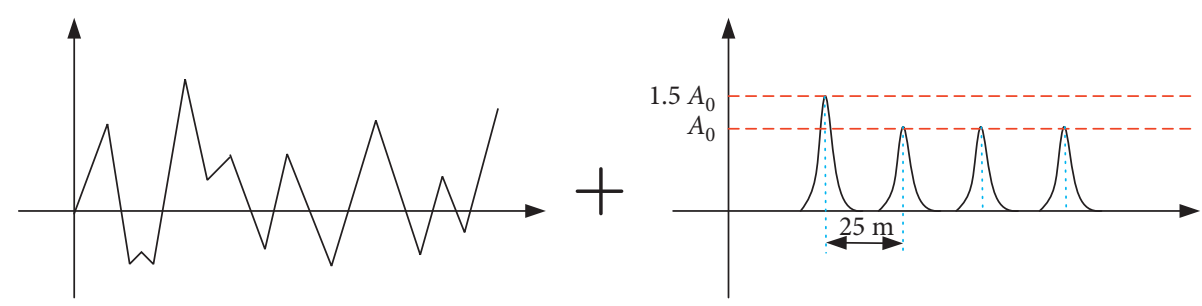

FIGURE 7: Illustration of rail irregularity.

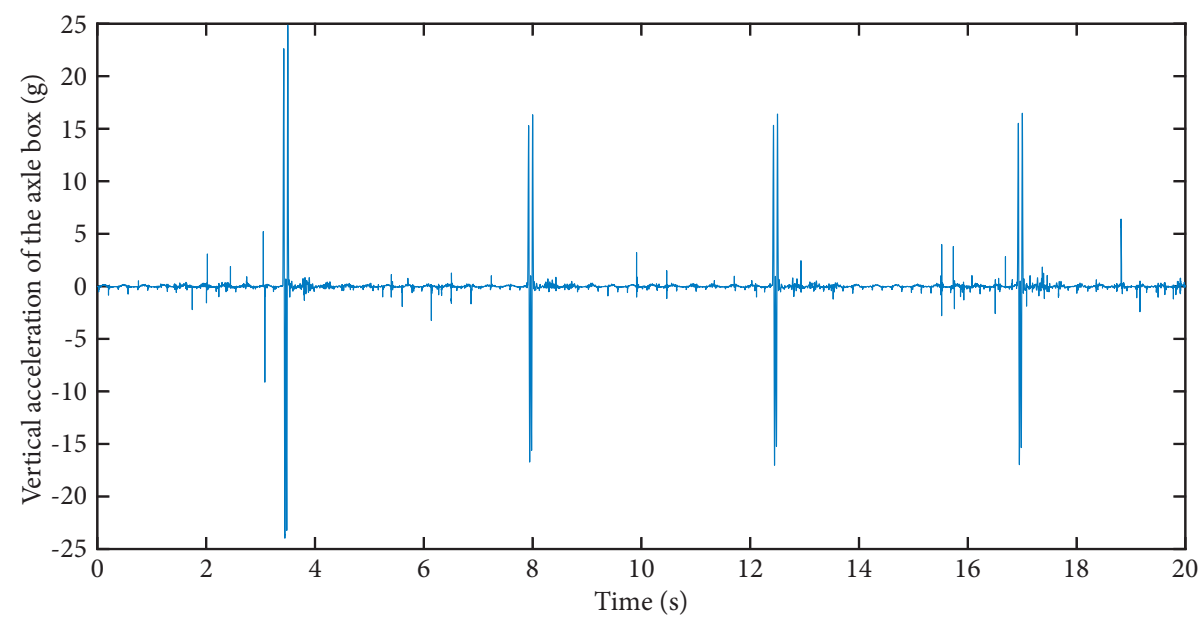

FIgURE 8: Vertical acceleration of the axle box during the whole experimental process.

energy of 181.4. The latter three impacts at $7.964 \mathrm{~s}, 12.470 \mathrm{~s}$, and $16.960 \mathrm{~s}$ were relatively weak,and also reached the peak at $20 \mathrm{~Hz}$, with the maximum instantaneous energy of 93.51, 92.71, and 106.20, respectively.

The aforementioned four impact positions and the data within a stationary comparison range $(0.2 \mathrm{~s})$ were selected for HHT. The integrals of any instantaneous frequency relative to time were calculated, and the marginal spectra were plotted for characterizing the total amplitude of various instantaneous frequencies, as shown in Figure 13. The main frequency of the impact position was $20 \mathrm{~Hz}$, while the main amplitude of the four impact points was 3.2799, 2.1433, 2.2127 , and 2.2496, respectively. The main amplitude of the signal within the stationary range was 0.0310 , which was far smaller than that within the impact range.

The quality indexes were then calculated based on the amplitudes at various impact points, as listed in Table 4 .

\section{Field Test}

4.1. Test Application Case 1. In case 1, the tramcar operated under the no-load condition at an operating speed of $40 \mathrm{~km} /$ h. Figure 14 shows the vertical acceleration data of the axle box in some abnormal vibration range during the operation. Apparently, some obvious impact positions were observed from the acceleration image in the range, thereby inducing abnormal vibration of the tramcar. The impacts appeared at an interval of approximately $2.3 \mathrm{~s}$ since $1.9 \mathrm{~s}$. The vertical acceleration of the axle box at the impact positions far exceeded the values in the stationary range. On account of seamed railways in this range, it was preliminarily judged from the interval of impact points that the impacts were induced by irregularities at the rail gap. The characteristic frequencies of abnormal vibration and the related causes were analyzed in detail as follows. 

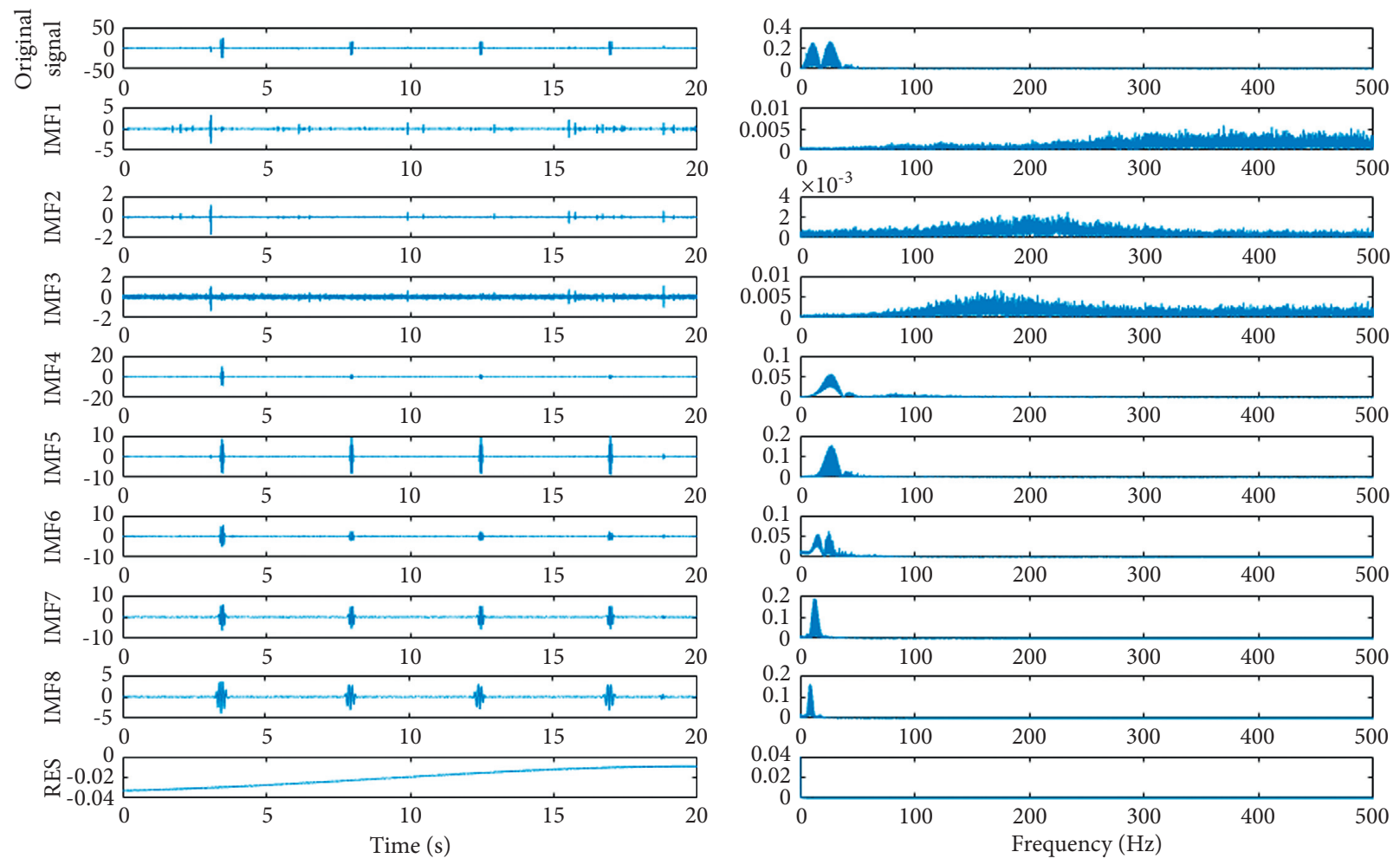

Figure 9: Time-domain and frequency-domain images of various components decomposed from the abnormal vibration signal via CEEMDAN, in which IMF1-IMF8 represent the first eight IMF components from high to low frequency, and RES represents the residual component.

TABLE 3: Calculated correlation coefficients between various IMF components of the abnormal vibration signal and the original signal.

\begin{tabular}{lc}
\hline Component & Correlation coefficient \\
\hline IMF1 & 0.0650 \\
IMF2 & 0.0293 \\
IMF3 & 0.0204 \\
IMF4 & 0.0458 \\
IMF5 & 0.1489 \\
IMF6 & 0.2741 \\
IMF7 & 0.1907 \\
IMF8 & 0.1380 \\
IMF9 & 0.2598 \\
IMF10 & 0.3663 \\
IMF11 & 0.2575 \\
IMF12 & 0.0256 \\
IMF13 & 0.0100 \\
IMF14 & 0.0179 \\
IMF15 & 0.0100 \\
IMF17 & 0.0366 \\
IMF18 & 0.0041 \\
&
\end{tabular}

4.1.1. CEEMDAN and HHT Analyses on the Abnormal Vibration Signal. First, the abnormal vibration signal of the axle box of the tramcar was decomposed via CEEMDAN, and 15 IMF components (denoted as IMF1-IMF15) from high to low frequency were obtained. The original signal, the first eight main IMFs, the residual component (RES), and the related frequency spectra could be plotted, as shown in Figure 15.
The correlation coefficients between various IMF components and the original signal were calculated, as shown in Table 5. Apparently, the IMF1-IMF5 components highly correlated with the original signal and could be regarded as the main components of the original vibration signal. The IMF1-IMF5 components, with the most significant characteristics of the original signal, imposed the greatest effect on the overall response. The correlation coefficients between IMF6-IMF16 components and the original signal were all less than 0.1 , which could thus be regarded as noise components or trend-term components. The IMF6-IMF16 components, with no significance to the feature extraction of the original signal, were eliminated.

Figure 16 compares the reconstructed signal from IMF1-IMF5 components with the original signal. Apparently, the denoised signal almost fitted well with the original signal, which retained the main characteristics of the main signal.

Next, after instantaneous processing on IMF1-IMF5 components of the vertical vibration signal, instantaneous frequency, instantaneous phase, and instantaneous energy could be obtained and the Hilbert spectra of the vibration signal could thus be plotted, as shown in Figure 17(a). The signal energy was almost distributed within the frequency band of $50-100 \mathrm{~Hz}$, while energy was relatively weak in the other frequency bands. The concentrated amplification of energy could be observed at around $2.0 \mathrm{~s}, 4.3 \mathrm{~s}, 6.6 \mathrm{~s}$, and $8.9 \mathrm{~s}$, and the instantaneous energy at these moments far exceeded the values at other moments. Four obvious impacts were observed during the whole vibration process. Figure 17(b) shows the Hilbert 


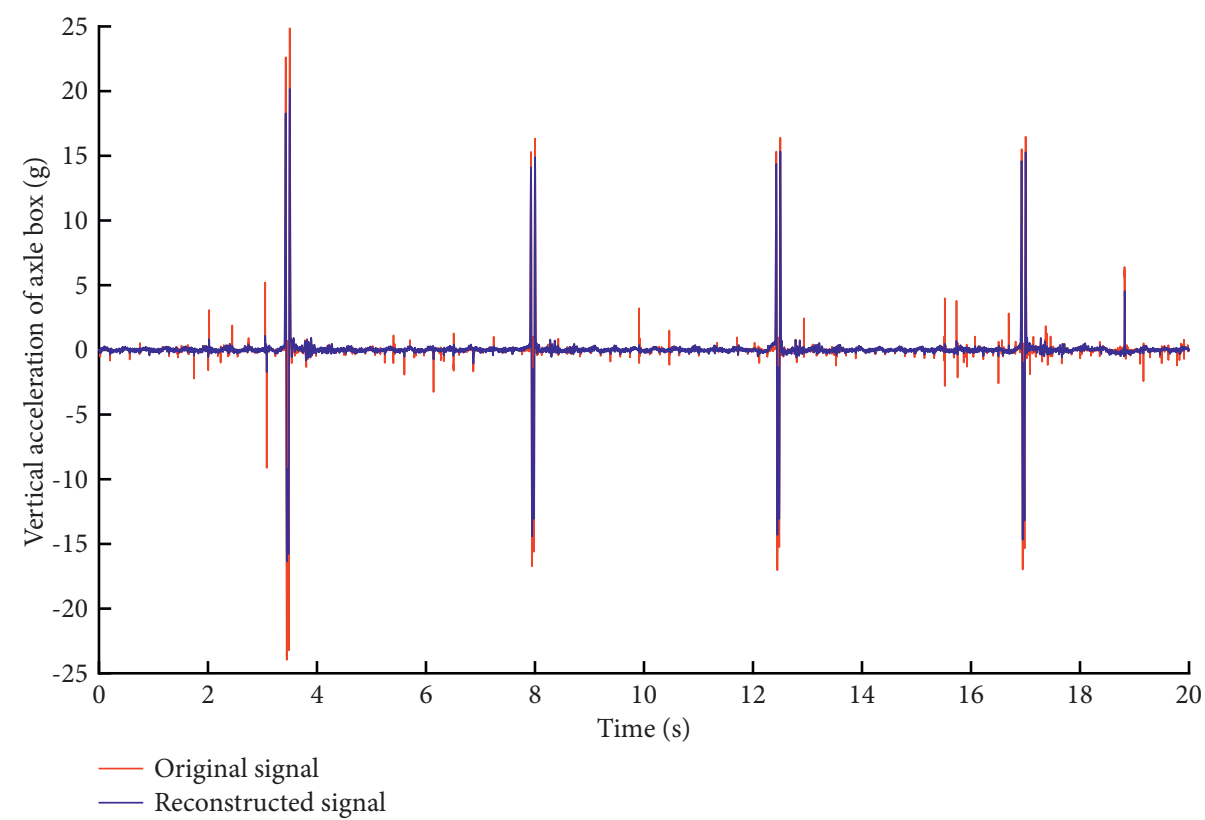

Figure 10: Comparison between the original and reconstructed signals.

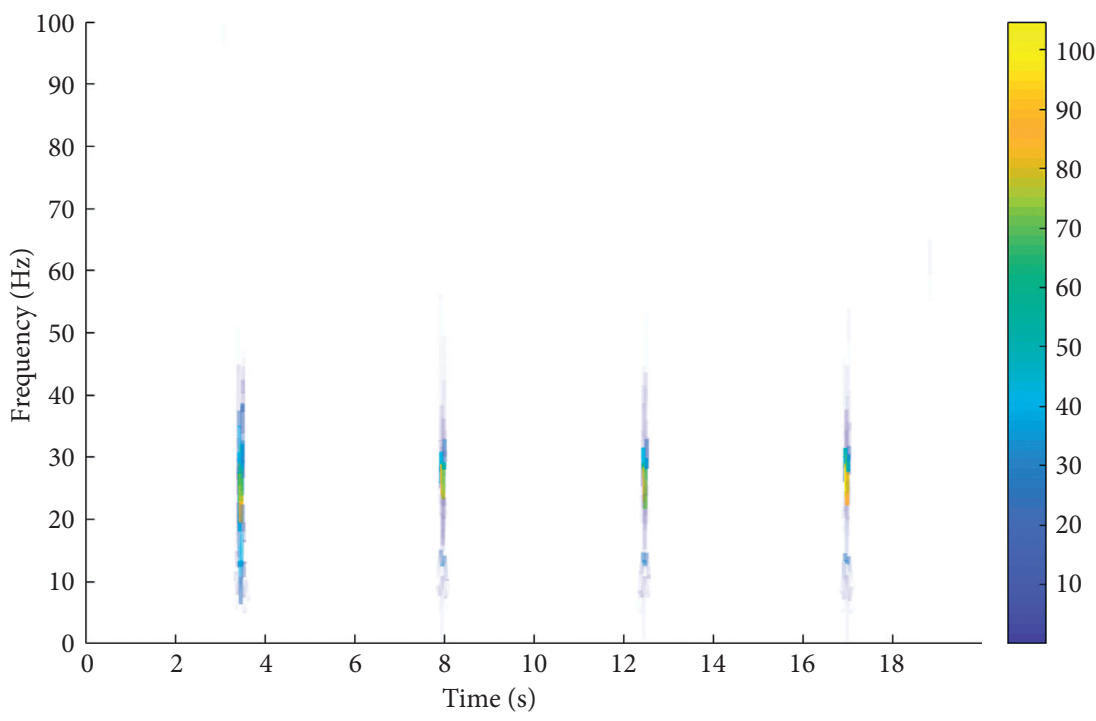

Figure 11: Hilbert spectrum of the abnormal vibration signal.

spectra using EMD-based HHT. As stated earlier, mode aliasing easily appeared during the decomposition of the railway impact signal with EMD; various components overlapped with each other, and a significant number of noise components were mixed in the signal and could hardly be eliminated. Accordingly, the Hilbert spectra at the impact moments showed a too wide frequency band. The energy was distributed within the whole frequency band of 0-200 Hz, which was unfavorable for individually analyzing the vehicle vibration frequency excited under rail impact. Therefore, abnormal vibration characteristics could not be adequately extracted. Comparing Figures 17(a) and 17(b), the proposed CEEMDAN-HHT showed better performance than the traditional EMD-HHT in the analysis of rail irregularities.
Figure 18 visually displayed the time-instantaneous, frequency-instantaneous energy relation of the vibration signal. Four impacts existed in this range at an average interval of approximately $2.3 \mathrm{~s}$. Moreover, the maximum instantaneous energy of impact increased steadily and reached the peak at $8.86 \mathrm{~s}$ and $70 \mathrm{~Hz}$, with a maximum instantaneous energy of 31.25.

The ranges before and after four impact positions with a length of $0.2 \mathrm{~s}$ and the data within the stationary comparison range $(5.80-6.00 \mathrm{~s})$ were selected for $\mathrm{HHT}$. The marginal spectra of the impact points and the stationary range were plotted via the integral, as shown in Figure 19. The signal energy in the impact range was mainly distributed in the lowfrequency region at around $60 \mathrm{~Hz}$. The main vibration 


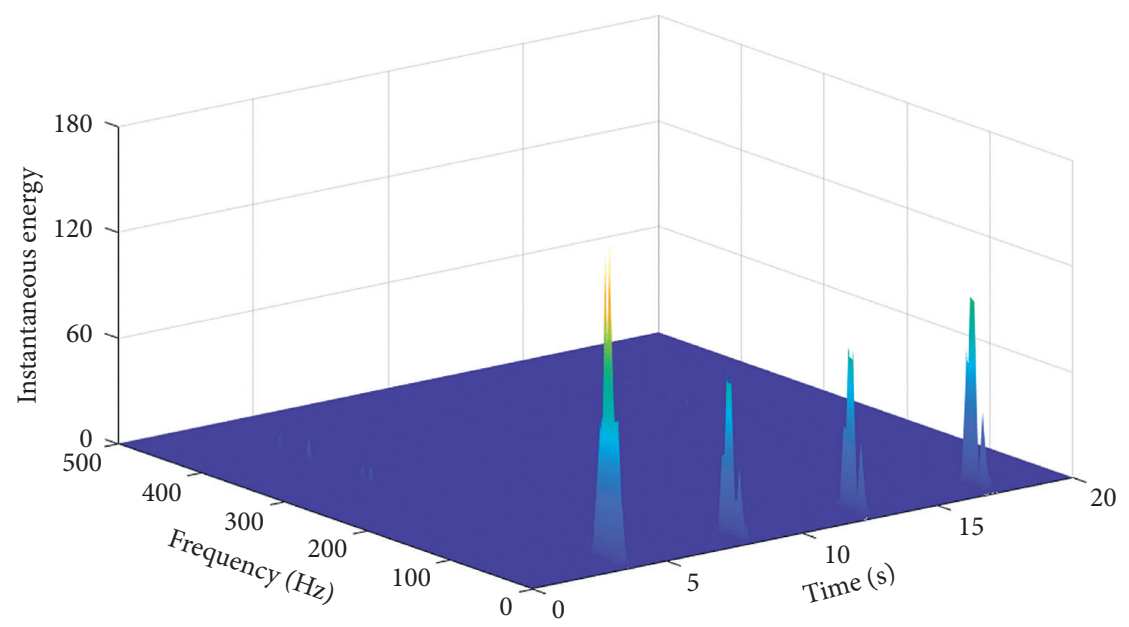

FIgURE 12: Time-frequency-instantaneous energy image.

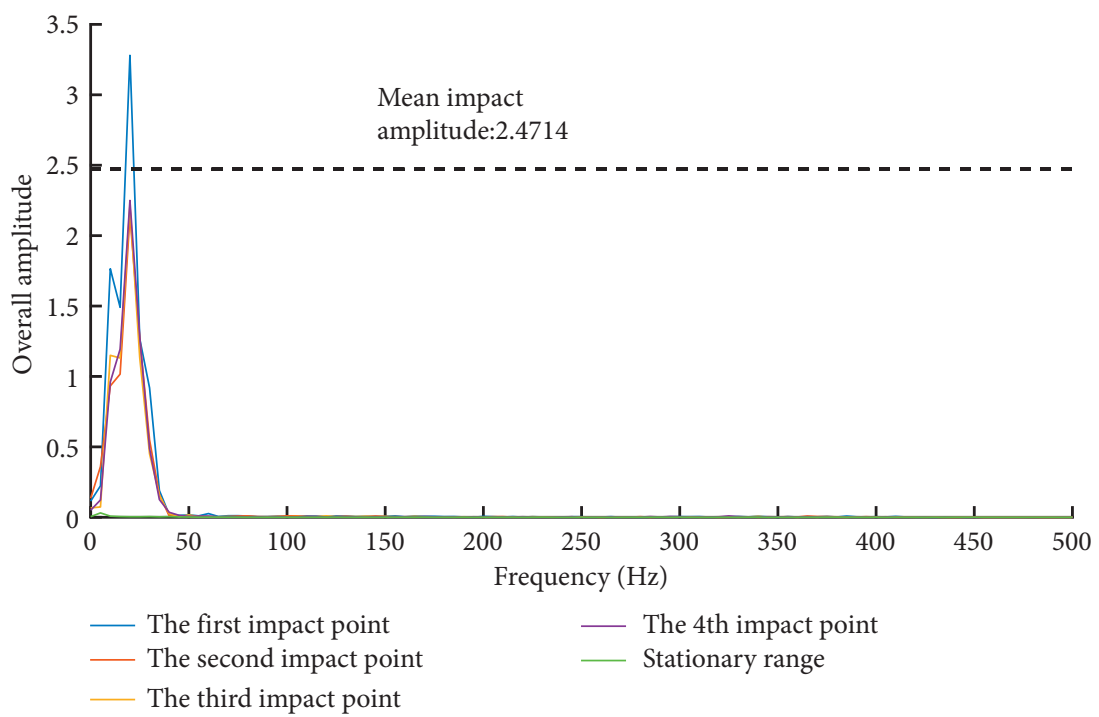

FIgURE 13: Marginal spectra of the abnormal vibration signal.

TABLE 4: Quality indexes at different impact points.

\begin{tabular}{lcccc}
\hline Impact position & First impact & Second impact & Third impact & Fourth impact \\
\hline Mass/Qu index & 1.3272 & 0.8672 & 0.8953 & 0.9103 \\
\hline
\end{tabular}

amplitude of the four impact signal was 0.0531, 0.1392, 0.0742, and 0.1965 , respectively. The signal energy in the stationary range was also mainly distributed in the low-frequency region at around $60 \mathrm{~Hz}$, and the main amplitude was 0.0344 , which was much smaller than the values at the impact points.

After preliminary analysis on frequency components, the following conclusions were drawn:

(1) The inherent frequency of $60 \mathrm{~Hz}$ was the bending frequency of the tramcar wheel set and also the main frequency when the tramcar operated under normal conditions.

(2) The vertical vibration acceleration in the impact range showed a concentrated amplification of energy at around $60 \mathrm{~Hz}$, with a frequency bandwidth of approximately $50 \mathrm{~Hz}$. Accordingly, the impact also triggered intensive resonance between vehicle and railway at a frequency of $60 \mathrm{~Hz}$.

The quality indexes at different impact points were calculated, as listed in Table 6. The quality indexes at the first and the third impact points were low, suggesting weak impacts; on the contrary, the impact at the fourth point was extremely large, indicating a strong abnormal impact.

4.1.2. Location of the Impact Points. Next, the rail line in the abnormal vibration segment was then surveyed. It was found that the line was laid by $25 \mathrm{~m}$ standard steel rails; that is, rail gaps existed after every $25 \mathrm{~m}$. Compared with the other 


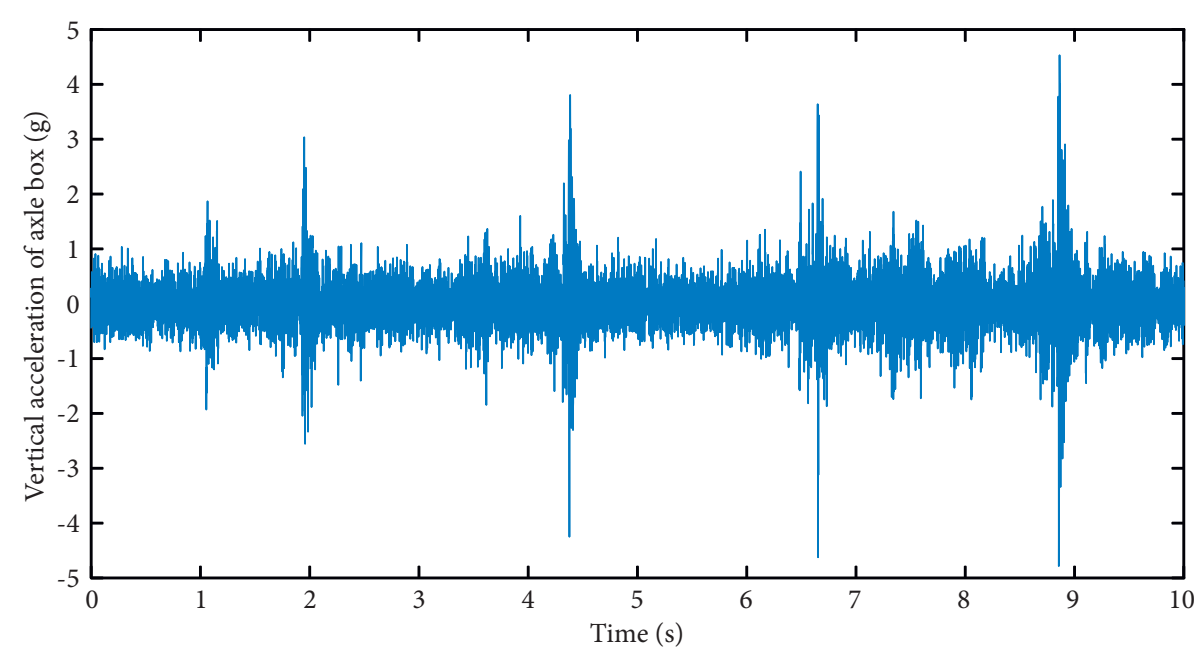

FIGURE 14: Vertical acceleration of the axle box during the whole test process.
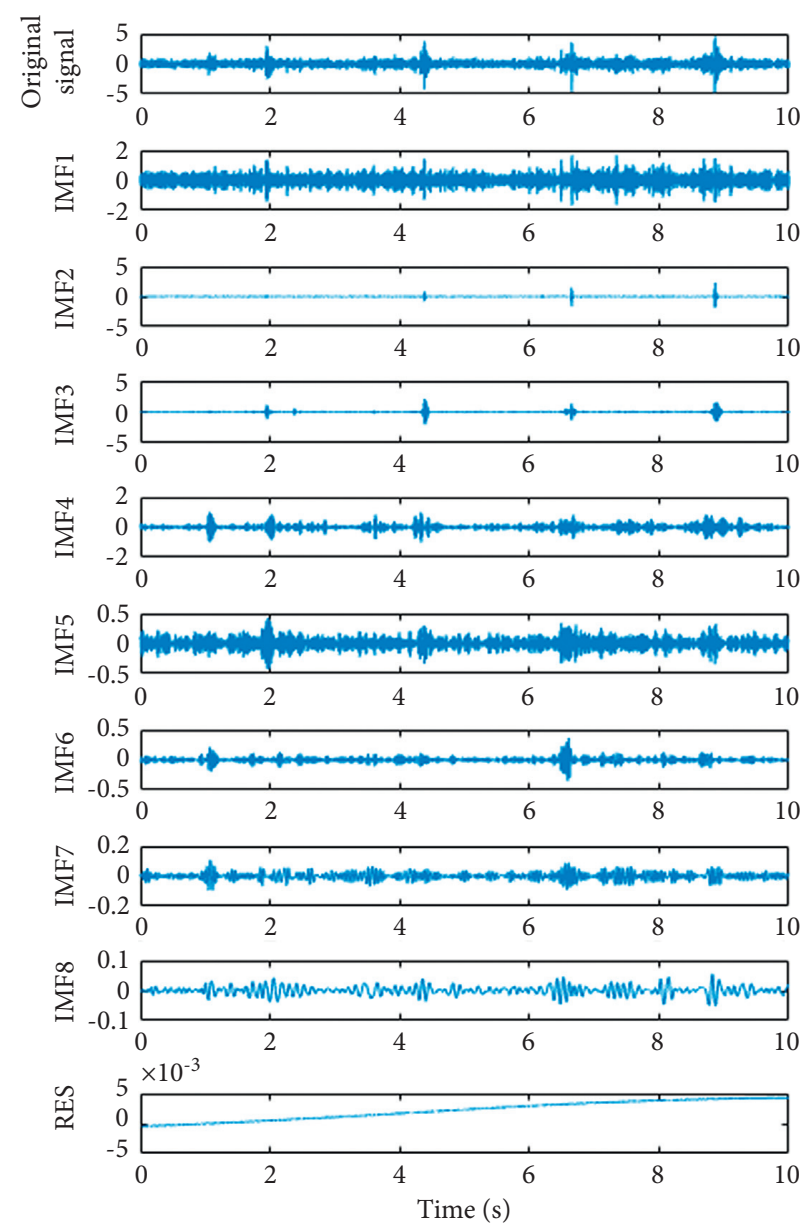
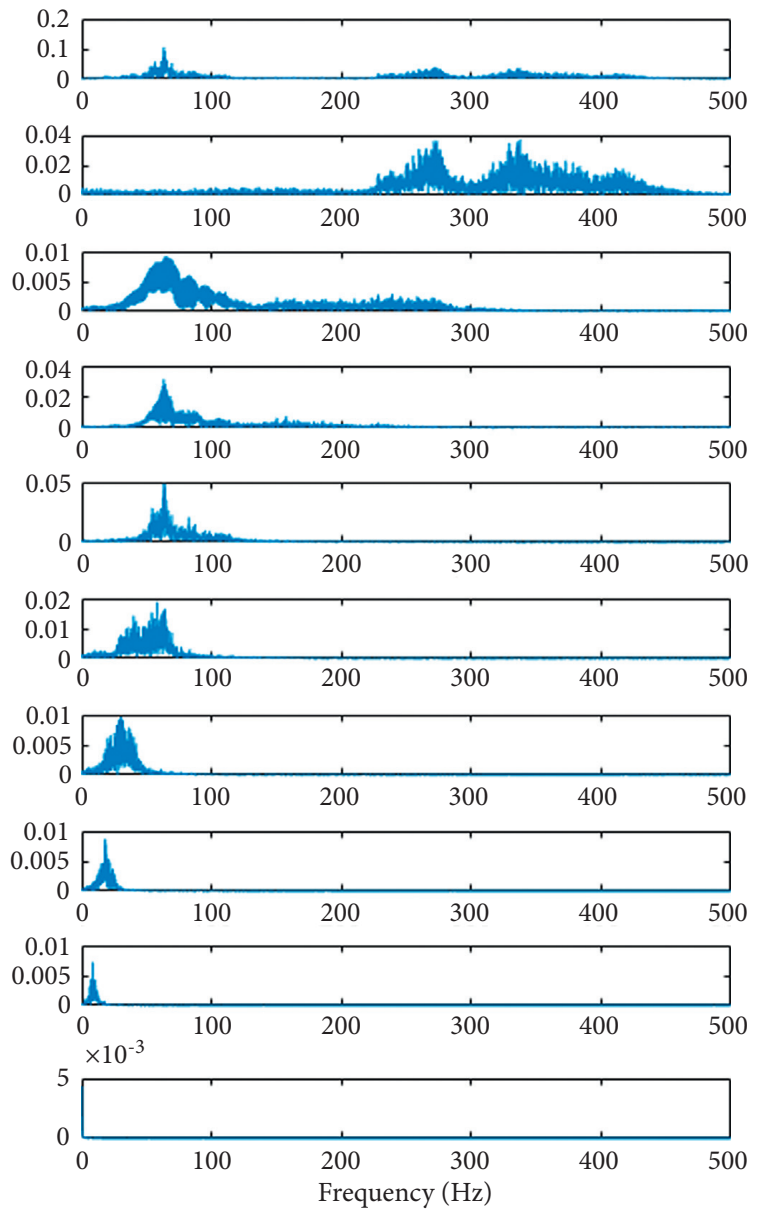

FIGURE 15: Time and frequency spectra of various components decomposed from the abnormal vibration signal via CEEMDAN.

segments, the junction plates were installed slightly above the rail, thereby producing impact between wheels and the joint plates during the operation of the tramcar. Through calculation, the tramcar at a speed of $40 \mathrm{~km} / \mathrm{h}$ passed through the rail gap at an interval of approximately $2.25 \mathrm{~s}$, and correspondingly, an impact was produced between rail and the joint plate at an interval of $2.25 \mathrm{~s}$, leading to a transient abnormal vibration. The actual phenomenon was in line with the aforementioned analysis results. By taking the integral relative to speed, the impact points could be located. Figure 20 shows an actual impact position on the line. The impact between the wheel and the top of the joint 
TABLE 5: Correlation coefficients between various IMF components of the abnormal vibration signal and the original signal.

\begin{tabular}{lc}
\hline Component & Correlation coefficient \\
\hline IMF1 & 0.7484 \\
IMF2 & 0.4031 \\
IMF3 & 0.3218 \\
IMF4 & 0.5173 \\
IMF5 & 0.3979 \\
IMF6 & 0.0993 \\
IMF7 & 0.0347 \\
IMF8 & 0.0041 \\
IMF9 & -0.0027 \\
IMF10 & -0.0047 \\
IMF11 & -0.0036 \\
IMF12 & -0.0023 \\
IMF13 & 0.0012 \\
IMF14 & -0.0014 \\
IMF15 & 0.0011 \\
\hline
\end{tabular}

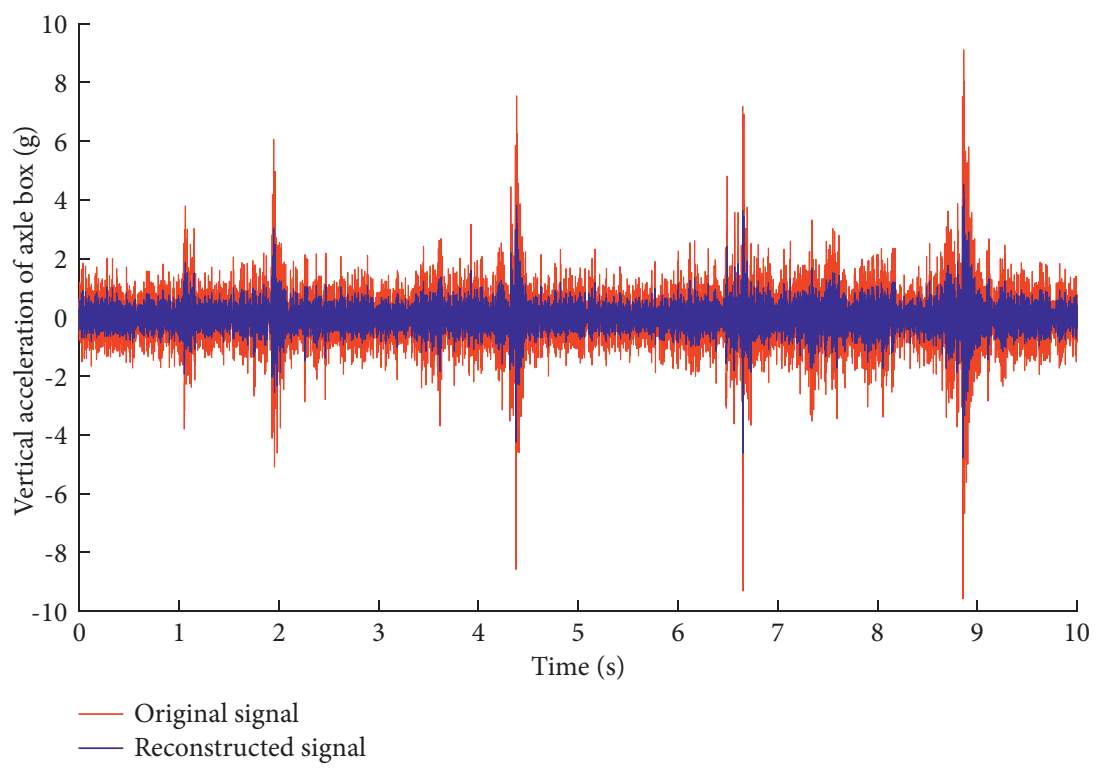

FIGURE 16: Comparison between original and reconstructed signals.

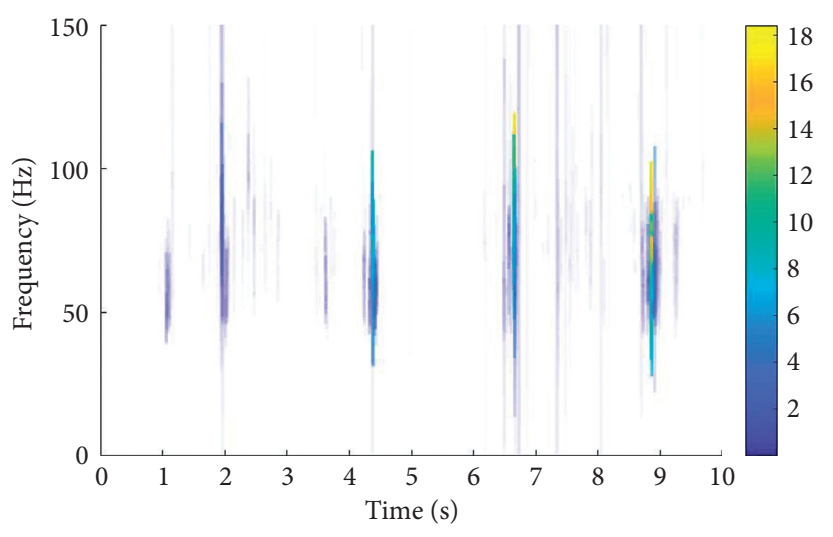

(a)

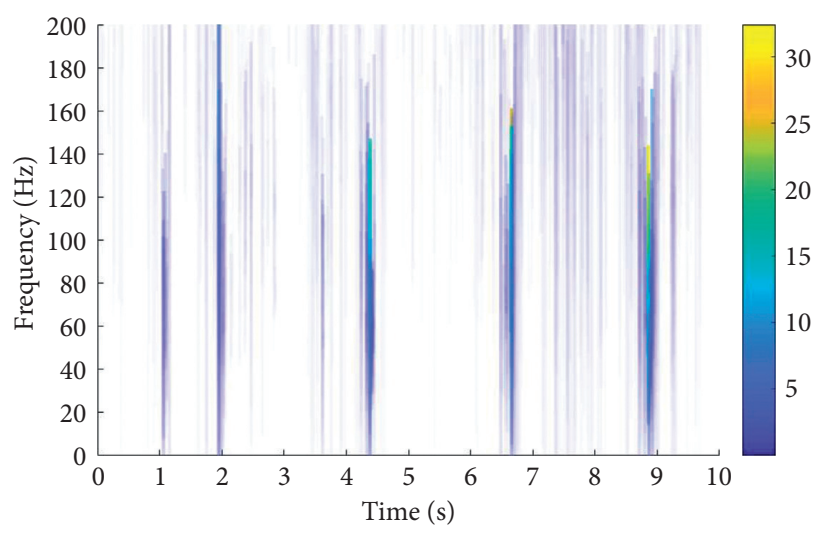

(b)

FIgURE 17: Hilbert spectra of the abnormal vibration signal: (a) Hilbert spectra after CEEMDAN-HHT and (b) Hilbert spectra after EMDHHT. 


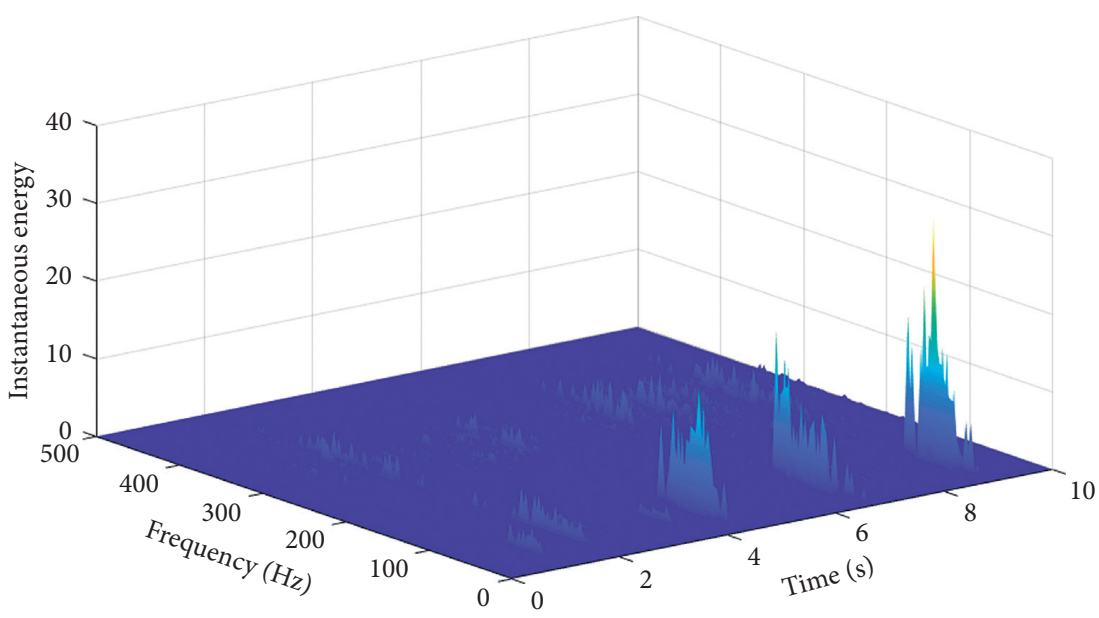

FIGURE 18: Time-instantaneous, frequency-instantaneous energy image of the abnormal vibration.

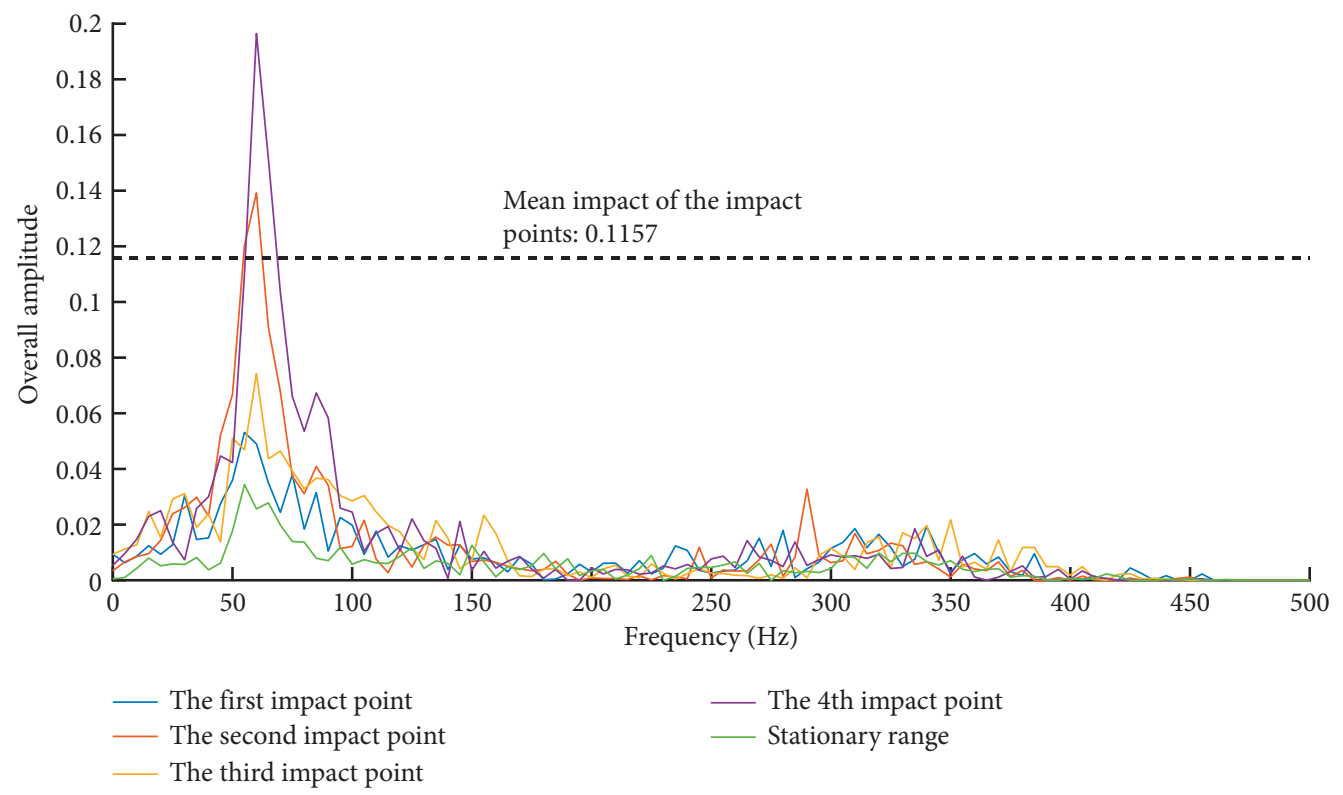

Figure 19: Marginal spectra of the vibration signal.

TABLE 6: Quality indexes of the impacts at different points.

\begin{tabular}{lcccr}
\hline Impact position & First impact point & Second impact point & Third impact point & Fourth impact point \\
\hline Quality index & 0.4589 & 1.2026 & 0.6410 & 1.6975 \\
\hline
\end{tabular}

plate at this position also excited the resonance between the tramcar and rail at a resonant frequency of $60 \mathrm{~Hz}$, accompanied with huge instantaneous energy in the impact segment.

4.2. Test Application Case 2. In case 2, the same tramcar was used, and serious flat damage was observed on the side wheels of the axle, as shown in Figure 21. When the vehicle traveled at a uniform speed, flat damage on wheels induced a periodic wheel-rail impact, thereby exciting periodic abnormal vibration of the axle box. The impacts were equivalent to those on the rail induced by periodic irregularities.
Therefore, the wheel flat damage was regarded as generalized rail irregularities. Next, the characteristic frequencies of abnormal vibration signals were extracted and investigated using the aforementioned method.

In case 2, the tramcar operated under AW0 no-load condition at a speed of $30 \mathrm{~km} / \mathrm{h}$. Figure 22 compares the vertical acceleration curves of the axle box between the flatscar wheel and normal wheel within $10 \mathrm{~s}$ during the normal operation process.

The acceleration signal of the axle box on flat-scar wheels was then decomposed with CEEMDAN, and 14 IMF components from high to low frequency were obtained. Figure 23 shows the original signal, the first eight main IMF 

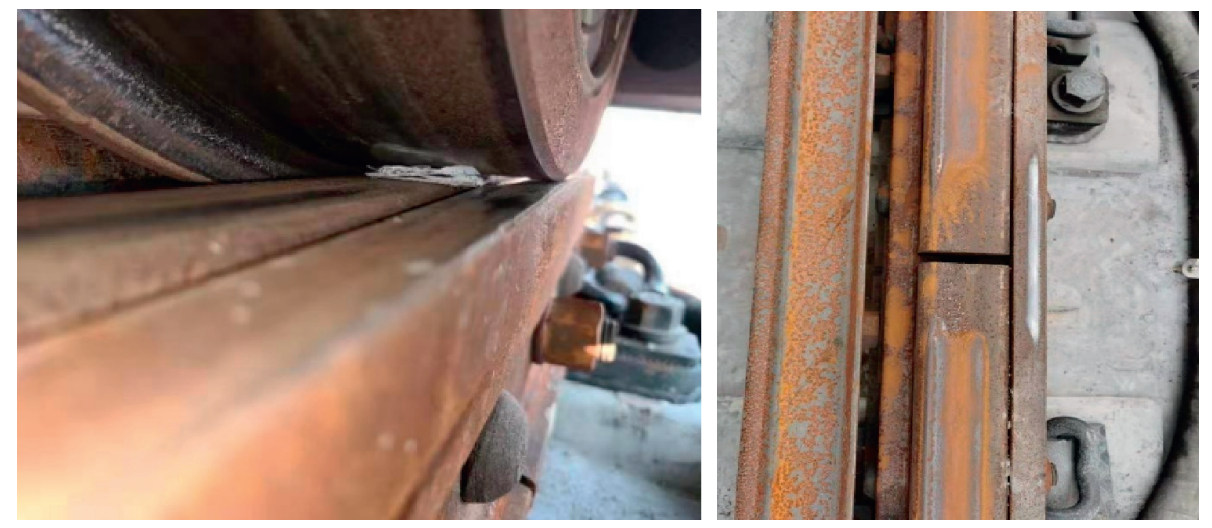

FIgURE 20: Impact on the top of the rail joint plate.

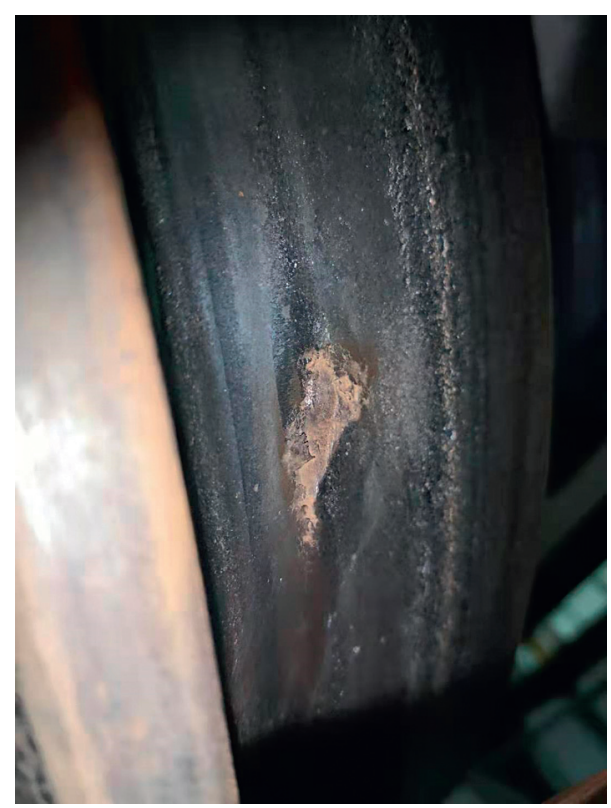

FIgURE 21: Flat-scar damage on the wheel.

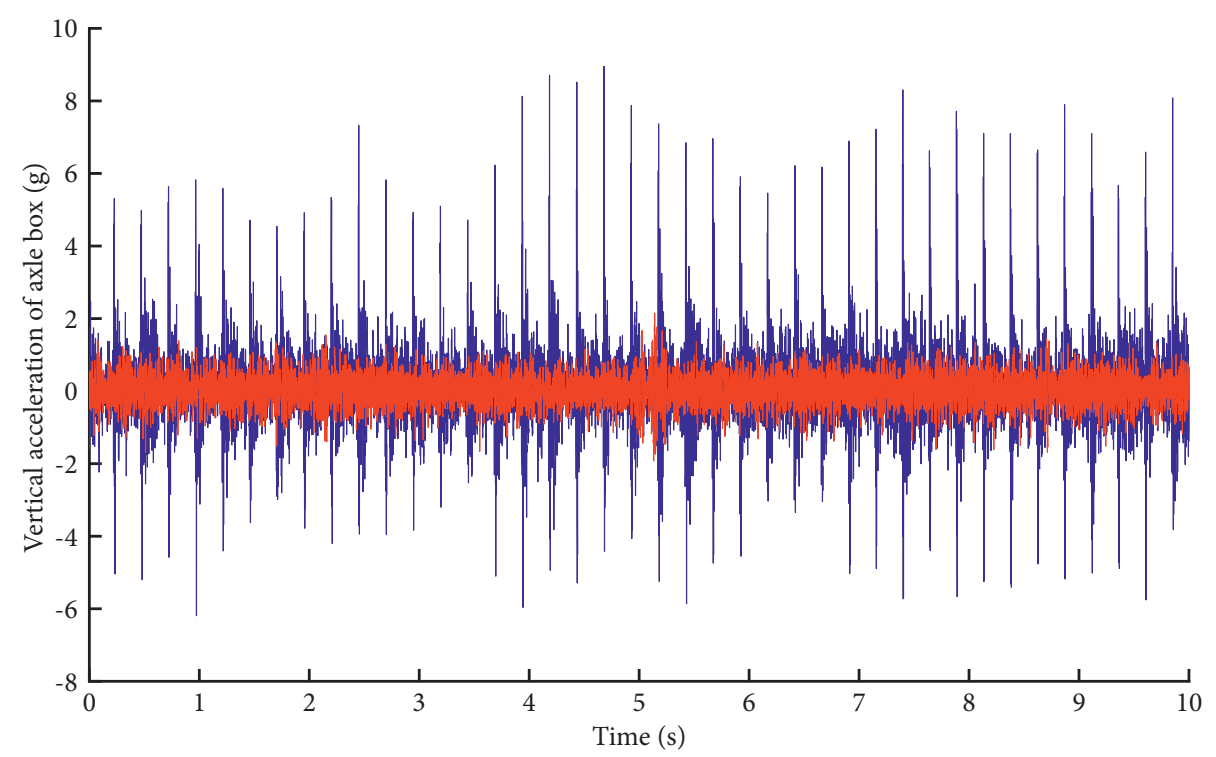

_ Flat-scar wheel

— Normal wheel

FIGURE 22: Comparison of the vertical acceleration of the axle box between flat-scar and normal wheels. 

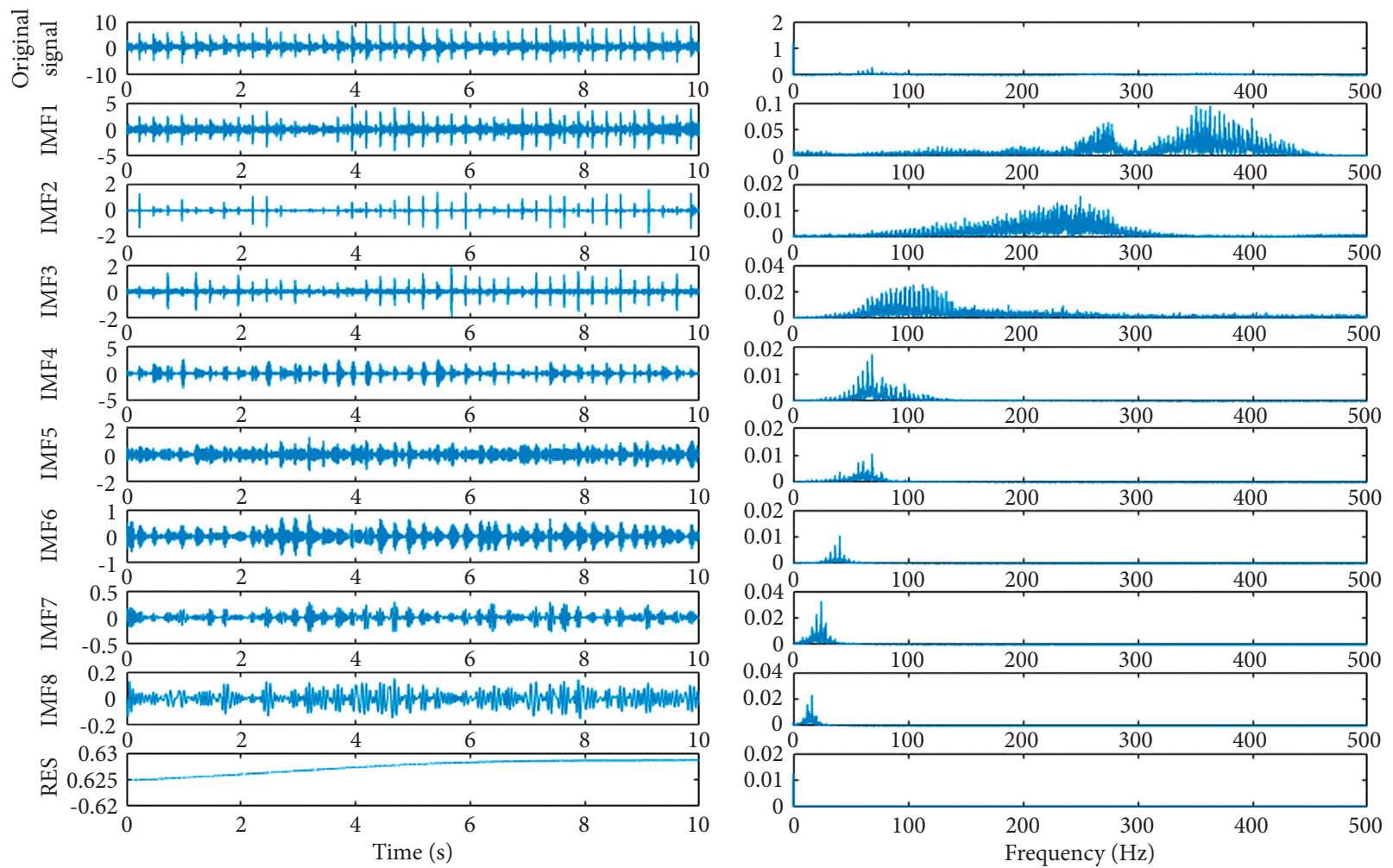

FIgURE 23: Time and frequency spectra of various components decomposed from the abnormal vibration signal via CEEMDAN.

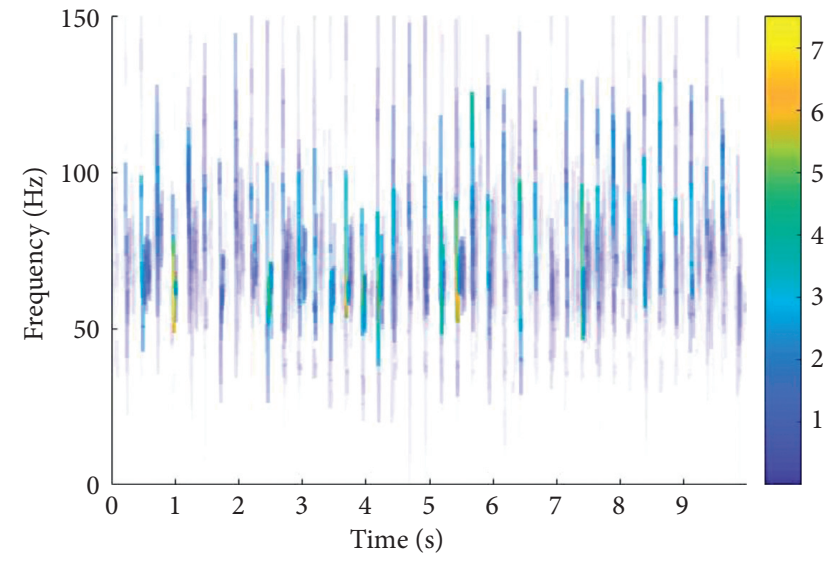

FIGURE 24: Hilbert spectra of the vibration signal of flat-scar wheels.

components, residual component (RES), and the related frequency spectra. The impact information on flat-scar wheels was mainly concentrated on IMF2, IMF3, and IMF4 components. The energy was concentrated at around $60 \mathrm{~Hz}$, with a vibration period of $0.25 \mathrm{~s}$.

After HHT on main information components of vertical vibration signal of the axle box, the Hilbert spectra were plotted, as shown in Figure 24. Overall, the signal energy was almost distributed in the frequency band of $50-100 \mathrm{~Hz}$. The periodic concentrated amplification of instantaneous energy at an interval of approximately $0.25 \mathrm{~s}$ was observed. Based on the time domain and Hilbert spectra, an intermittent impact disturbance was produced on the wheel-rail when the flatscar wheels traveled, thereby bringing about a periodic impact on the corresponding axle box. The maximum vertical acceleration on the axle box at the impact points was five times greater than the values in the stationary segment.

\section{Conclusions}

In view of the limitations of traditional time-frequency analysis methods such as Fourier transform in nonstationary and nonlinear signal processing, Hilbert-Huang transform (HHT) is applied to analyze the abnormal vibration of tram. Aiming at the problems of mode aliasing in the EMD method used in the classical HHT, this article proposes to decompose the original vibration data using the fully adaptive noise set empirical mode decomposition (CEEMDAN), then eliminate the trend-term component by the correlation coefficient method, and denoise the high-frequency noisy component using the wavelet threshold method and weighted reconstruction of the highfrequency component and low-frequency information component after denoising, Finally, the optimization process of Hilbert Huang transform is carried out. Taking a tram as the research object, the Hilbert spectrum of axle box vertical acceleration is obtained through HHT, the corresponding relationship of time-instantaneous frequency-instantaneous energy is obtained, and the impact point is located. Combined with the marginal spectrum, the vibration characteristics are analyzed, the quality index is calculated, and the abnormal vibration cause and fault mode of the tram are judged.

Based on the local characteristic time scale of signal, HHT decomposed complex signals into limited IMF components and performed feature extraction on various IMF components of the tramcar vibration signal, which could 
effectively identify both trend and state of abnormal vibrations, thereby laying a foundation for the identification of tramcar failure modes. The impact on the operating tramcar induced the corresponding inherent frequencies, which could thus lead to the change in the distribution of the instantaneous energy with instantaneous frequency. For a tramcar, the Hilbert spectra of the vibration signal showed a difference in impact and stationary segments, which could thus be used for abnormal vibration analysis and failure diagnosis of tramcars. HHT marginal spectrum could visually reflect the relationship between various frequencies and overall amplitude (overall energy) of the vibration signal of the tramcar, distinguish the impact and the stationary segments, and find the true frequencies of impact-induced abnormal vibration so as to judge the corresponding failure mode.

\section{Data Availability}

The data used to support the findings of this study are included within the article.

\section{Conflicts of Interest}

The authors declare that they have no conflicts of interest.

\section{Acknowledgments}

This project was supported by the Postdoctoral Innovation Talent Support Program (no. BX20200240), China Postdoctoral Science Foundation (no. 2020M671207), and the Natural Science Foundation of Shanghai (no. 21ZR1467100) and sponsored by the Shanghai Sailing Program (no. 20YF1451100), Shanghai Colabrative Innovation Research Center for Multinetwork \& Multimodel Rail Transit.

\section{References}

[1] L. Xu, W. Zhai, and J. Gao, "A probabilistic model for track random irregularities in vehicle/track coupled dynamics," Applied Mathematical Modelling, vol. 51, 2017.

[2] L. Xu and W. Zhai, "A novel model for determining the amplitude-wavelength limits of track irregularities accompanied by a reliability assessment in railway vehicle-track dynamics," Mechanical Systems and Signal Processing, vol. 86, 2017.

[3] J. Gao, W. Zhai, and Y. Guo, "Wheel-rail dynamic interaction due to rail weld irregularity in high-speed railways," Proceedings - Institution of Mechanical Engineers, vol. 232, no. 1, 2018.

[4] J. Xu, Study on Random Vibration Analysis of High Speed Vehicle Track Coupling System and Evaluation Method of Track Irregularity, Southwest Jiaotong University, Jiaotong, China, 2016.

[5] G. Yang, G. Li, and Z. Liu, "Vibration characteristics analysis of gearbox housing system of high-speed train subjected to wheel-rail excitation," Journal of the China Railway Society, vol. 39, no. 11, pp. 46-52, 2017.

[6] Z. Niu, Vibration Characteristics and Experimental Study of Axle Box System of High Speed Train, Jilin University, Jilin, China, 2019.

[7] X. Xiao, Z. Sun, and W. Shen, "A Kalman filter algorithm for identifying track irregularities of railway bridges using vehicle dynamic responses," Mechanical Systems and Signal Processing, vol. 138, 2020.

[8] Q. Xiao, Y. Cheng, and X. Xu, "Vibration characteristic analysis of high-speed train gear transmission system under track irregularity," Journal of Mechanical Transmission, vol. 45, no. 4, pp. 135-141, 2021.

[9] S. L. Grassie, "Measurement of railhead longitudinal profiles: a comparison of different techniques," Wear, vol. 191, no. 1, 1996.

[10] A. Caprioli, A. Cigada, and D. Raveglia, "Rail inspection in track maintenance: a benchmark between the wavelet approach and the more conventional Fourier analysis," $M e$ chanical Systems and Signal Processing, vol. 21, no. 2, 2005.

[11] M. Molodova, Z. Li, and A. Nunez, "Monitoring the railway infrastructure: detection of surface defects using wavelets," in Proceedings of the 2013 16th International IEEE Conference on Intelligent Transportation Systems: Intelligent Transportation Systems for All Modes: 2013 16th International IEEE Conference on Intelligent Transportation Systems, pp. 1316-1321, The Hague, The Netherlands, October 2013.

[12] X. Kang, L. I. Guoqing, W. Wang, and J. Liu, "Evaluation method for high-speed railway welded joints based on resonance demodulation technology," China Railway Science, vol. 32, no. 5, pp. 90-95, 2011.

[13] J. Liu, D. Chen, G. Zhao, and L. Liu, "Track impact index method for evaluating track short wave irregularity of high speed railway," China Railway Science, vol. 37, no. 4, pp. 34-41, 2016.

[14] S. Chen, J. Lin, and J. Chen, "Dynamic characteristics extraction of vehicle-track vertically coupling system based on improved EMD," Journal of Vibration and Shock, vol. 30, no. 8, pp. 212-216, 2011.

[15] Z. Li, S. Lian, and X. Liu, "Time-frequency analysis of vehicletrack vertically coupling system based on hilbert-huang transform," Journal of Vibration, Measurement \& Diagnosis, vol. 33, no. 05, pp. 799-803+911, 2013.

[16] P. Su, Y. Chen, and W. Yan, "Time-frequency analysis of bridge vibration based on HHT method," Earthquake resistant engineering and Retrofitting, vol. 41, no. 4, pp. 160-166, 2019.

[17] Y.-x. Li and L. Wang, "A novel noise reduction technique for underwater acoustic signals based on complete ensemble empirical mode decomposition with adaptive noise, minimum mean square variance criterion and least mean square adaptive filter," Defence Technology, vol. 16, no. 3, pp. 543-554, 2020.

[18] W. Chen, J. Li, Q. Wang, and K. Han, "Fault feature extraction and diagnosis of rolling bearings based on wavelet thresholding denoising with CEEMDAN energy entropy and PSOLSSVM," Measurement, vol. 172, Article ID 108901, 2021.

[19] M. K. Babouri, N. Ouelaa, T. Kebabsa, and A. Djebala, "Diagnosis of mechanical defects using a hybrid method based on complete ensemble empirical mode decomposition with adaptive noise (CEEMDAN) and optimized wavelet multiresolution analysis (OWMRA): experimental study," International Journal of Advanced Manufacturing Technology, vol. 112, no. 9-10, pp. 2657-2681, 2021.

[20] F. Lin, R. Wang, Y. Yang, Q. Xiao, Z. Xu, and X. Dong, "Analysis on the influence of flat spot on wheel-rail impact," Machine Tool \& Hydraulics, vol. 49, no. 3, pp. 140-144, 2021.

[21] S. G. Newton and R. A. Clark, "An investigation into the dynamic effects on the track of wheelflats on railway vehicles," Journal of Mechanical Engineering Science, vol. 21, no. 4, 2006.

[22] T. Wang, M. Zhang, Q. Yu, and H. Zhang, "Comparing the applications of EMD and EEMD on time-frequency analysis of seismic signal," Journal of Applied Geophysics, vol. 83, 2012. 